\title{
Proposed EEG measures of consciousness: a systematic, comparative review
}

\author{
André Sevenius Nilsen*, Bjørn Erik Juel, Benjamin Thürer, Johan Frederik Storm* \\ Brain Signalling Group, Department of Molecular Medicine, Institute of Basic Medical
}

Sciences, University of Oslo (UiO), 0372 Oslo, Norway

*Corresponding authors at: Department of Physiology, Institute of Basic Medical Sciences, University of Oslo; Sognsvannveien 9, 0372 Oslo, Norway; Tel.: +47 90804477 and +47992 95 763, Email addresses: sevenius.nilsen@gmail.com and j.f.storm@medisin.uio.no

\begin{abstract}
Objective measures capable of distinguishing between conscious and unconscious states are important for improving both clinical decisions and our fundamental understanding of consciousness. Here, we present a systematic review of 255 electroencephalography (EEG)-based measures of consciousness that have been proposed and tested in humans in the past decade, and provide detailed overviews of general categories and specific measures of consciousness. We show that measures based on signal diversity and event-related potentials are the most consistent in how they change in unresponsive states compared to wakefulness. Specifically, spectral entropy (SpecEnt), Lempel Ziv complexity (LZc), and spectral edge frequency (SEF95), seem to be the most practical, consistent, and reproducible. However, since most studies did not collect current or retrospective subjective reports from participants, but inferred the state of consciousness from (lack of) behavior, the presence or absence of consciousness in conditions of unresponsiveness is uncertain. This limits the conclusions that can be drawn until our knowledge regarding consciousness in unresponsive states improves.
\end{abstract}

Keywords: consciousness, unconsciousness, responsiveness, unresponsiveness, EEG, anesthesia, disorders of consciousness, sleep 


\section{HIGHLIGHTS}

- We review and compare 255 EEG based measures of consciousness

- Measures based on signal diversity or event-related potentials track unresponsiveness

- $\quad$ LZc., SpecEnt., and SEF95, are practical, consistent, and generally reproduced

- This review may provide a basis for future evaluation of measures of consciousness

\section{INTRODUCTION}

Consciousness research has attracted an increasing number of prominent neuroscientists in the last decades, but the field is still maturing and lacks consensus about even the general form of the solutions to the field's most basic questions (Albright et al., 2000; Crick \& Koch, 1990; Doerig et al., 2020; Michel et al., 2019; Seth, 2010). One reason why consciousness has been difficult to pin down for scientific study is the fact that the term "consciousness" can take on distinct meanings in different contexts. For example, assessing a patient's capacity to respond coherently to stimuli (i.e. responsiveness) is often used for judgment about the patient's state of consciousness in clinical settings (e.g. as is implicit in the Glasgow Coma Scale; Sternbach, 2000, and the Modified Observer's Assessment of Alertness/Sedation Scale; Chernik et al., 1990), but this is insufficient if the target is the more basic notion of consciousness as phenomenological, subjective experience, as described by Nagel (1974) (see Box 1 for a clarification of terms). In many cases, phenomenal consciousness can be present completely independently of responsiveness (Sanders et al., 2012). Hence, in order to assess the presence of phenomenal consciousness in individuals who are unable to communicate, measures based on brain activity are needed.

In the past few decades, a large number of methods and measures have been proposed and tested for this precise purpose (e.g. Engemann et al., 2018; Gaskell et al., 2017; Haenggi et 
al., 2009; Höller et al., 2014; Mediano et al., 2018; Sitt et al., 2014; Stefan et al., 2018). Unfortunately, in most of the studies where the efficacy of such measures has been tested, the researchers appear to have relied on traditional, practical criteria for consciousness (i.e. responsiveness), or on preconceived notions about whether a specific condition (e.g. general anesthesia) is associated with conscious experience or not (e.g. Ni Mhuircheartaigh et al., 2013). However, even if the original studies did not strictly determine the actual state of consciousness in their test subjects, knowledge of how proposed measures behave in and across different conditions can be useful as our collective understanding of the general state of consciousness in those conditions improve. Thus, a review of the large and growing list of proposed measures of consciousness may shed light on which measures can be considered strong candidate measures of consciousness per se. Additionally, since the literature on proposed measures of consciousness is large and unwieldy, it is a formidable task for the uninitiated to obtain a good overview of which measures exist and might be promising.

With this review, we provide a systematic overview of EEG-based measures of consciousness that have been tested and used in the past 10 years (2009-2018). We believe such a review may aid the search for better measures of consciousness in the years to come. Such measures, if also theoretically well-grounded, might then even be used to infer the presence of consciousness in situations where reports are in principle unavailable, such as during early development and in non-human organisms (Boly et al., 2013; Lavazza \& Massimini, 2018). In addition, while there are studies comparing specific measures across various physiological states (e.g Casarotto et al., 2016; Schnakers et al., 2008) or studies comparing several measures on specific states (e.g. Gosseries et al., 2011; Höller et al., 2014), no reviews to our knowledge comprehensively compare measures across conditions. Crucially, if we are to develop a general measure of consciousness, then such a measure should be independent of 
pharmacological, physiological, or psychological state, as long as it reliably tracks the presence or absence of consciousness in individuals. Thus, we aim to review proposed measures of consciousness not only based on the amount of evidence behind a given measure (i.e. reproducibility) for specific conditions, but to also review how this measure performs across conditions (i.e. consistency).

\section{BOX 1. Consciousness vs. responsiveness}

As the field of consciousness research is riddled with terms that have no unique, agreed-upon definition, we provide a brief clarification of how certain terms are to be understood in this review.

Consciousness: The term consciousness is here meant to refer to phenomenal, subjective experience itself, or "what it is like to be" (Nagel, 1974). Consciousness in this sense does not require behavior or ability to communicate, even though behavior and communication typically reflect consciousness during normal wakefulness in humans. This is a main reason why behavioral (un)responsiveness is typically used to assess the level of consciousness in patients (e.g. Sternbach, 2000).

Responsiveness: Responsiveness is defined here as a state with presence of non-reflexive, goal-directed behavior and/or complex and coherent responses to sensory stimuli, including the ability to communicate verbally or non-verbally. However, being unresponsive does not entail being unconscious (Sanders et al., 2012). For example, there are several physiological, pharmacological, and pathological states that are known to be associated with both complete behavioral unresponsiveness and conscious experience, such as dreams 
(Chennu et al., 2013; Leslie et al., 2007; Nielsen, 2000; Noreika et al., 2011a; Sanders et al., 2012; Siclari et al., 2018). Thus, measures of consciousness, in the sense of phenomenal, subjective experience should not depend on behavior.

Measure of consciousness: Here, we define a measure of consciousness to be a quantitative measure based on objective observations (e.g. recording of brain activity) with the capacity to distinguish between conscious and unconscious states, without relying on behavior. Although a measure of consciousness would, in principle, have the capacity to discriminate between states with and without any kind of phenomenal experience, most studies aimed at assessing states of consciousness from the last decades only assessed the responsiveness of the subjects. Even though assessing the presence of consciousness could be done by inferring from immediate or delayed subjective reports, even the most immediate subjective reports do not provide indubitable proof of consciousness - they may, for example, result from confabulation or may be distorted by memory limitations (Schwitzgebel, 2008). Thus, one might even question if it will ever be possible to strictly confirm or falsify any measure of consciousness.

\subsection{Current state in the field of EEG measures of consciousness}

While behavioral, positive markers of certain aspects of consciousness (such as the Glasgow coma scale; Sternbach, 2000; or the mirror test; Gallup et al., 2002) are useful in some situations, research has shown the need for measures of consciousness that are independent of behavior (Casali et al., 2013; Massimini et al., 2009; Vanhaudenhuyse et al., 2018). As it is 
widely agreed that some parts of the cortico-thalamic system constitute the main substrate associated with normal human consciousness (e.g. Dehaene et al., 1998; Koch et al., 2016, but see Merker, 2007), it seems reasonable that electroencephalography (EEG), which provides a direct readout of neocortical, electrical activity at millisecond resolution (Buzsáki et al., 2012), is well suited as a basis for an objective measure of consciousness (Koch et al., 2016). In addition, since the equipment needed for EEG is relatively inexpensive, easy to use, and mobile, it is applicable in a broad range of situations where misclassification of consciousness is common (Leslie, 2017; Schnakers et al., 2009). Given these advantages of EEG, it is not surprising that a large number of EEG-based potential measures of consciousness have been proposed.

Studies that examine potential EEG-based measures of consciousness come from various scientific fields including medical clinical science (e.g. Gosseries et al., 2011), neuroscience (e.g. Sarasso et al., 2015), and basic physical sciences (e.g. Fekete et al., 2018). This has led to the parallel development of potential EEG measures from different groups. Some of those measures have mostly been investigated as anesthesia monitors (e.g. Bispectral index; BIS; (Duchateau et al., 2014; Shepherd et al., 2013) whereas others have been primarily used on disorders of consciousness (DOC) patients (e.g. late responses to auditory stimuli; Bekinschtein et al., 2009). Some measures require specific equipment or know-how (e.g. perturbational complexity index; PCI; Casali et al., 2013) while others require only standard EEG equipment that is available to many labs and clinics (e.g. Amplitude Coalition Entropy; ACE; Schartner et al., 2015). Overall, the wide range of properties makes straightforward comparison among alternatives difficult. 
While no broad overview of EEG-based measures of consciousness exists to date, there have been several previous efforts to compare and combine several measures on the same data material (see e.g. Engemann et al., 2018; Gaskell et al., 2017; Haenggi et al., 2009; Höller et al., 2014; Mediano et al., 2018; Sitt et al., 2014; Stefan et al., 2018). However, these comparisons were performed either in a limited number of conditions or with a limited number of candidate measures. In other studies, researchers discussed possible neural correlates of consciousness more generally and did not focus specifically on EEG-based methods for practical use (Koch et al., 2016; Rees et al., 2002; Seth et al., 2008).

While it is difficult to directly compare the individual alternative measures (e.g. due to a wide variety of types and implementations of available measures), getting an overview of the efficiency of broad categories of measures could be useful for guiding future developments. For example, in the last decade, two broad categories have received much attention: measures of signal diversity, complexity, or entropy (e.g. Fan et al., 2011; Schartner et al., 2015; Shalbaf et al., 2013) and measures of functional or effective connectivity (e.g. Chennu et al., 2016; Mashour \& Hudetz, 2018; Shin et al., 2016). These categories of measures have to a certain extent been inspired by theoretical considerations (Sergent \& Dehaene, 2004; Tononi, 2004) as well as capturing electrophysiological properties of brain activity that have for long appeared to be connected to changes in consciousness (e.g. low amplitude, fast, asynchronous EEG activity in wakefulness vs high amplitude, slow, coherent activity in states like deep sleep and general anesthesia; Rees et al., 2002). Despite recent advances, time-frequency-based measures that have been used for nearly a century to study anesthesia and sleep (Hughes \& John, 1999; Loomis et al., 1937) are still widely used today (e.g. Koch et al., 2016; Warnaby et al., 2017), and measures capturing cortical responses to sensory 
stimuli are commonly employed in the clinic (Engemann et al., 2018; Luauté et al., 2010). For more details on these categories, see sections 2.2 and 3.1.2-3.1.6.

In sum, despite a large and diverse set of proposed EEG-based measures of consciousness and efforts to compare and contrast them, a comprehensive and systematic review comparing and evaluating these measures is missing from the literature.

\subsection{Aim}

This review provides a comprehensive overview, evaluation, and categorization of potential EEG-based measures of consciousness that have been tested and used in the past 10 years (publication years: 2009-2018). In addition, we aim to highlight knowledge gaps and possible uncertainties in the current literature, such as results that have not yet been replicated, which may motivate future empirical studies.

By using a systematic approach, this review strives to evaluate the literature as objectively as possible based on the reported results and not the authors' interpretations of their own results. However, while the general conditions of general anesthesia, sleep, and DOC, are commonly used as exemplars of states of unconsciousness, we stress that in most of the studies covered in this review the presence or absence of phenomenal experience was not explicitly investigated through for example use of immediate or delayed subjective reports (but see Schwitzgebel, 2008, for a discussion on the limits of introspection). Thus, we do not in this review make any assumptions or conclusions about whether a certain condition, such as ketamine or propofol anesthesia, should be treated as a state of unconsciousness, but assume that the state of (un)consciousness within a particular condition is comparable between studies (see section 4.1.1 for a discussion). Such an objective approach allows the readers to compare the proposed EEG measures within and across conditions and to draw their own conclusions 
relevant to their contexts. In other words, this review does not give any simple answer to the question of which is the "best" measure of consciousness, but provides an overview of how different (categories of) measures change in specific conditions, relative to wakefulness, which might be useful as our understanding of consciousness in these conditions improve.

\subsection{Structure of the review}

First, we provide an overview of the systematic review methodology (section 2), with further details to be found in the supplementary material (Suppl. 1-4). Next, we provide an overview and comparison of the main categories of EEG-based measures of consciousness (section 3.1). Finally, we provide an overview and comparison of a selection of individual EEG-based measures of consciousness (section 3.2). For easy access and readability, the main text contains only summary tables and references used in-text, as the tables containing the individual measures, categories, and references, are too large for print. These main tables are provided as three supplementary data sheets (denoted SDAT) with information about how to utilize the supplementary data in Suppl. 5. A list of abbreviations used in the text and tables are presented in Box 2.

\section{BOX 2. Abbreviations}

For an expanded description of some of the terms abbreviated here, see Suppl. $6 . \Delta$ : change caused by the filtering process, i.e. change from before (S1.2) to after (S1.6) filtering; AAI: a-line autoregressive index; AEP: auditory evoked potential; Anesthesia: all forms of anesthesia in which the patient is unresponsive; ApEn: approximate entropy; BIS: bispectral index; CI: consistency index (described in Suppl. 3); Connectivity: functional and effective connectivity based measures; DOC: disorders of consciousness; DR: dream report; EMG: electromyography; ERP: event-related potential (ERP) based measures; IFT: isolated forearm technique; HV: high variance; LZc: Lempel Ziv complexity; MCS: minimally conscious state; Mix / Other: anesthetics not mentioned (e.g. xenon, nitrous oxide) or a mix of several anesthetics, "unpredictability", or "stochasticity", based 
measures; MLAEPi: middle latency auditory evoked potential index; Multi-parameter: combination of several types of measures; NA: not applicable; $\mathbf{N}_{\mathbf{M}}$ : number of unique measures; $\mathbf{N}_{\mathbf{s}}$ : number of unique studies; NREM: stage 1-4 sleep; O: other comments; Other: measures not fitting in other categories; PeEn: permutation entropy; PCI: perturbational complexity index; PG: patient groups; REM: rapid eye movement sleep; ROC: return of consciousness following anesthesia; S1.0-S1.6: filtering steps (described in Suppl. 1); SDAT: supplementary data; SEF95: spectral edge frequency (95th percentile); Signal diversity: signal variability/predictability based measures; Sleep: all stages of naturally occurring sleep; SpEn: spectral entropy; Suppl.: supplementary material; Time-frequency: frequency decomposition based measures; UWS: unresponsive wakefulness syndrome.

\section{METHODS}

\subsection{Systematic literature search and objective evaluation}

We performed a literature search in Scopus (https://www.scopus.com) to find all original articles that used the term "conscious*" combined with "electroencephalo*" or "eeg" in their title, abstract, or keywords (last search date: 04.12.2018). The resulting 5719 original articles were filtered according to a priori defined inclusion and exclusion criteria. Our inclusion criteria were defined as: original, empirical studies, published less than 10 years prior to our final search (01.01.2009 - 04.12.2018), and using at least one of the terms "consciousness", "awareness", or "vigilance". Exclusion criteria were defined as: non-human studies (e.g. exclusively computational, theoretical, or animal studies), single-case studies, perception studies, meditation studies, epilepsy studies, and conference proceedings. The remaining 140 studies were carefully reviewed to produce the first, raw overview of proposed EEG-based measures of consciousness. For a full list of references to all included studies, see supplementary data (SDAT: Supplementary_References). 
For the first overview, summarized in the "Raw Table" (SDAT: Supplementary_Measures, marked "S1.0", see also Figure 1), the main results of each study were recorded, one for each condition that was investigated. We only included studies with a wake control state, and, for each measure in each condition, we note whether there was a significant increase $(+)$, decrease (-), or no significant change $(=)$ in the measure, relative to the wakefulness condition. We did not investigate changes between unresponsive states such as NREM and REM sleep since we cannot infer the state of consciousness in these conditions (see section 4.1.1 for a discussion). Thus, we summarized how each measure changed in each condition relative to the awake control condition without further evaluating the validity of the measure relative to an assumed ground truth about consciousness. In order to better characterize measures that behave differently when calculated on different frequency bands we decided to split measures accordingly (see S1.0 for details, and section 4.1.3 for a discussion).

\section{[FIGURE 1]}

To condense the table and make it more informative, we applied several predefined steps (Figure 1; details can be found in Suppl. 1). First, measures that were named differently, but appeared similar upon inspection (e.g. renormalized partial directed coherence; Maksimow et al., 2014, and partial directed coherence; Höller et al., 2014), were merged and treated as a single measure as long as this did not introduce inconsistencies (S1.1). Then, we performed an extended literature search on each EEG measure from the original search to prevent removing valuable measures based on insufficient data (S1.2). Next, we filtered out measures that were not reproduced (S1.3), had inconsistencies within conditions (S1.4), or that were not reproduced in multiple conditions (S1.5). Finally, we noted technical comments about the 
measures that were reported in the reviewed articles which might affect the measure's validity as a measure of consciousness (S1.6)

\subsection{Categorization and evaluation of measures}

To give an overview of the different classes of measures that appeared in our search, each measure was allocated to one of six specific categories and summarized. While there are many potential ways to group different measures, we here employed commonly used distinctions (see section 1.1). Category names are italicized to distinguish when a term is used to denote a category and when the same term is used to denote the underlying concept. The six categories were: connectivity, signal diversity, event-related potential (ERP), time-frequency, multi-parameter, and other.

The category connectivity contains measures that estimate the functional or effective connectivity between brain regions, such as granger causality and phase lag index. Signal diversity contains measures that quantify the variability/predictability of the EEG signal, such as approximate entropy (ApEn) and Lempel-Ziv complexity (LZc). ERP contains measures based on the analysis of characteristics of EEG responses to sensory stimuli or perturbations through for example transcranial magnetic stimulation (TMS), such as auditory evoked potentials (AEP) and blink related (de)synchronization. Time-frequency includes measures that are based on information in the time-frequency decomposition of the EEG signal, such as bispectral index (BIS) and frequency band power. Multi-parameter contains measures that are based on several individual EEG measures that were combined to form a composite index, such as cerebral state index and detector of consciousness. Other contains the remaining EEG measures that did not fit in any of the other categories, such as analysis of microstates and intrinsic network reactivity index. 
Some measures might however be difficult to classify. For example, BIS captures multiple aspects of the time-frequency domain and combines them to an overall index, thus being both a time-frequency and multi-parameter measure. In these situations, a measure was classified as multi-parameter only if it was based on different domains (e.g. ERPs and time-frequency).

The categorized measures were aggregated across individual measures resulting in a category table, which was updated at four different steps of the 6-step literature procedure ("S1.2", “S1.3”, "S1.4”, “S1.6”). For further details, see supplementary material (Suppl. 2).

We evaluated each category on two aspects:

1) Robustness of a category, here measured by how many individual parameters and studies were left after the 6-step process outlined earlier (Figure 1), relative to the initial and expanded literature search. Specifically, robustness is defined as the change in number of studies (absolute and relative terms); $\Delta N_{S}=N_{S, S 1.6}-N_{S, S 1.2}\left(\%: 1-N_{S, S 1.6} / N_{S, S 1.2}\right)$, and the number of measures; $\Delta N_{M}=N_{M, S 1.6}-N_{M, S 1.2}\left(\%: 1-N_{M, S 1.6} / N_{M, S 1.2}\right)$, from before (S1.2) to after (S1.6) the filtering process. Thus, robustness here indicates to what extent the individual categories are self-consistent and sufficiently reproduced within and between conditions.

2) Consistency of a category, here measured by how consistent the directionality of effects are within and between conditions. Specifically, we established a consistency index $(C I)$ which is the ratio between "+” and “_" signs, normalized by the sum of all effect directions, and weighted by the total amount of results (see Suppl. 3). Consistency thus indicates to what extent the different measures within the category results in the same direction of change (up, down, none) relative to wake controls. As such, consistency can not only be used as a metric of the construct reliability of a specific category but also to indicate if different parameters within a category are to some extent interchangeable. It is important to note that a low $C I$ 
score does not mean that a category is "bad", it rather suggests that the low $C I$ category needs further investigation, or is sensitive to specifics of the implementation of measures, or is too inclusive (e.g. connectivity might be split in directed and undirected connectivity), or that different measures within that category might show different effect directions in different conditions.

Individual measures were evaluated on their level of reproduction (i.e. whether they were tested in all conditions, multiple times) and consistency/reliability using $C I$ (i.e. consistency of effect directionality). In addition, individual measures were evaluated based on aspects of practicality (i.e. general applicability). Finally, during the review process, some measures were found to show "counterintuitive" results for certain conditions (e.g. a measure might not return to pre-anesthesia levels after reemergence from anesthesia). These results were noted, however, we did not perform an expansive search to find such "contraindications".

It is important to note that $C I$ is here intended to provide a) additional information (overall consistency of effect direction), b) a way to order different measures, and, c) a summary metric of the distribution of results. Thus, given a heterogeneity in the mechanisms underlying the various conditions, differences in implementation of measures (technical, methodological, analytical), differences in recording setup (sampling rate, referencing, etc.), and variations in exactly which parts of the signal a measure is applied to (regional or global selection of channels, frequency band, etc.), the value of the $C I$ is dependent on the information one is interested in and is not meant as a valuation metric in terms of how "good" or "promising" a measure or category is. 


\section{RESULTS}

\subsection{Promising Categories of EEG measures of consciousness}

We will first discuss the category table as a whole, before (S1.2) and after (S1.6) the filtering process (see section 3.1.2), then go through each category in detail (see sections 3.1.3 - 3.1.7), before summarizing the results (see section 3.1.8). We focus primarily on how the categories fare with respect to their robustness and consistency (see section 2.2). However, we will also show how the consistency of categories are affected when split into frequency bands (note that frequency band-specific results are extrapolated, see Suppl. 4). For the purpose of discussion and presentation of results, we have grouped the different conditions together in anesthesia, DOC, and sleep, rather than providing results for each sub-condition (e.g. keeping the sleep condition split into NREM and REM conditions).

\section{[TABLE 1]}

\section{[FIGURE 2]}

\subsubsection{Results for categories of measures}

Table 1 presents the summarized overview of all categories after each main step in the filtering process (see Figure 1). The full table, including results for every sub-condition, can be seen in the supplementary data (SDAT: Supplementary_Measures and SDAT: Supplementary_Categories). Figure 2 presents a graphical overview of the spread of measures within each category according to consistency and effect direction, both before (at S1.2; light grey color) and after the filtering process (at S1.6; colored). 
Initially, when judging the "Extended Table" (S1.2 in Table 1), four categories have a relatively high number of publications: connectivity $\left(N_{S}=61\right), \operatorname{ERP}\left(N_{S}=67\right)$, signal diversity $\left(N_{S}=83\right)$, and time-frequency $\left(N_{S}=129\right)$. Similarly, the same categories stand out in terms of the number of measures and the total number of results. The categories other and multi-parameter are naturally less populated since measures within these categories are relatively unique and often not used outside of a few select groups. In terms of overall consistency across conditions, measures, and implementations, only three categories have a $C I>0.5: \operatorname{ERP}(C I=0.60)$, signal diversity $(C I=0.75)$, and multi-parameter $(C I=0.92)$.

In the "Final Table" (S1.6 in Table 1), following the filtering process, the overall pattern is similar as before the filtering process, with the following major changes: the category multi-parameter is no longer represented as these measures has only been tested within anesthesia, and that the category $E R P$ has seen a large increase in consistency after removal of non-reproduced and inconsistent measures $\left(C_{\mathrm{S} 1.6}-C I_{\mathrm{S} 1.2}=0.20\right)$. In terms of robustness, the following categories were most to least affected by the filtering process: multi-parameter $\Delta N_{S}$ $=-10(-100 \%), \Delta N_{M}=-4(-100 \%)$, connectivity $\Delta N_{S}=-32(-52.46 \%), \Delta N_{M}=-27(-75 \%)$, other $\Delta N_{S}=-3(-27.27 \%), \Delta N_{M}=-3(-75 \%)$, time-frequency $\Delta N_{S}=-18(-13.95 \%), \Delta N_{M}=-20$ $(-69.43 \%), E R P \Delta N_{S}=-13(-19.40 \%), \Delta N_{M}=-18(-69.23 \%)$, and signal diversity $\Delta N_{S}=-23$ $(-27.71 \%), \Delta N_{M}=-18(-54.55 \%)$

\section{[TABLE 2]}

Finally, we looked at the $C I$ distribution across frequency bands (Table 2), using an extrapolation method described in Suppl. 4, in order to investigate if certain frequency bands were more consistent in their effect direction across all conditions. A highly consistent frequency band would indicate that all conditions affect that band in a similar way within a 
measurement category. For example, if functional connectivity in the delta band is seen to increase for all measures of functional connectivity, then the delta band would have a high $C I$ for the category connectivity. By performing a weighted average (by number of results) across all categories, we can see if a specific frequency band is generally consistent. Here, we see that the overall estimated consistency before the filtering process ( $\mathrm{S} 1.2$ in Table 2) is relatively equal among the bands: $\delta C I=0.41, \boldsymbol{\alpha} C I=0.43, \boldsymbol{\beta} C I=0.42$, and $\boldsymbol{\gamma} C I=0.47$, with $\boldsymbol{\theta} C I=0.28$ and $\mathbf{b b C I}=0.62$ being the least and most consistent frequency bands, respectively. After the filtering process (S1.6 in Table 2), CI increased across the board except in alpha; $\left(\Delta C I=C I_{\mathrm{S} 1.6}-C I_{\mathrm{S} 1.2} ; \boldsymbol{\delta} \Delta C I=0.17, \boldsymbol{\theta} \Delta C I=0.09, \boldsymbol{\alpha} \Delta C I=-0.02, \boldsymbol{\beta} \Delta C I=0.09, \boldsymbol{\gamma} \Delta C I=0.01\right.$, and $\mathbf{b b} \Delta C I=0.08)$, with $\boldsymbol{\theta} C I=0.37$ and $\mathbf{b b} C I=0.71$ the least and most consistent frequency bands, respectively.

In sum, the categories signal diversity and ERP were both relatively consistent $(C I>0.60)$, had a relatively high number of studies $\left(N_{S}>54\right)$ and measures $\left(N_{M}>8\right)$, and were among the most robust to the 6 -step filtering process $\left(\Delta N_{S}>72.29 \%, \Delta N_{M}>30.77 \%\right)$. In terms of frequencies, broadband and delta band was the two most consistent bands $(\boldsymbol{\delta} C I=0.58, \mathbf{b b C I}$ $=0.71)$, after the filtering process, across all categories.

\subsubsection{Connectivity}

Measures within the category connectivity aim to describe and quantify the functional or effective integration between spatially segregated brain areas. In this paper, the category is mostly based on functional connectivity analysis of spontaneous EEG data (e.g. granger prediction; Kim et al., 2012, or Directed Transfer Function; DTF; Bertini et al., 2009).

While the category as a whole has relatively low consistency $(C I=0.20$ at $\mathrm{S} 1.6)$, this may be explained by the heterogeneity in measures. Measures like global field synchrony capture 
global phase synchronization (e.g. Nicolaou et al., 2014), while mutual information captures how much information about one EEG channel is contained in another (e.g. Thakor \& Tong, 2004). Furthermore, measures that capture directed connectivity often show opposite results when comparing anterior-to-posterior to posterior-to-anterior (e.g. Maksimow et al., 2014), long-range connectivity compared to short-range local connectivity (e.g. Rizkallah et al., 2019), and whether connectivity is measured in the high or low frequency bands (e.g. Lee et al., 2019). For example, we see that consistency varies from $C I=0.01$ for the delta band, to $C I=0.39$ for the gamma band and $C I=0.37$ for broadband before the filtering process (see Suppl. 4). After the filtering, broadband was the most consistent with $C I=0.52$, with delta next, with $C I=0.30$. This change is primarily driven by the consistency of mutual information $\left(C I=0.75\right.$ at S1.6), which carries $\sim^{2 / 3}$ of the results of the delta band, and $\sim 1 / 3$ for the broadband.

So, while connectivity as a category is not very consistent across measures and frequencies, the picture is not so clear within conditions. Both before and after the filtering process, connectivity seems to typically be reduced in anesthesia and DOC, while mostly increasing during sleep. However, after the filtering process, the dominant increase in connectivity in sleep is caused by the measure global field synchrony (GFS). Treating GFS as an outlier would nearly double the consistency in the "Final Table". Thus, for connectivity-based measures, it might be more informative to treat the measures individually, rather than as a category. Furthermore, connectivity-based measures differ in whether they indicate local or global connectivity, directed front-to-back or back-to-front connectivity, etc. Therefore, some measures might introduce apparent inconsistencies because of merging. While not merging connectivity measures could more accurately reflect the heterogeneous nature of measured changes in connectivity, this would decrease robustness as many measures would not be 
reproduced unless merged (see section 4.1 .3 for a discussion on merging). Finally, while it has been suggested that a certain type of structural connectivity is necessary (but not necessarily sufficient) for a system to be conscious (e.g. Mashour et al., 2020), functional connectivity does not accurately reflect structural connectivity (for a discussion see Das \& Fiete, 2020).

\subsubsection{ERP}

The category ERP includes EEG measures that are derived from sensory event-related potentials (e.g. Auditory Event-related Potentials; AEP; Yppärilä et al., 2004) and potentials evoked by physically induced perturbations (e.g. TMS-evoked potentials; Massimini et al., 2009). Thus, this category contains a diverse range of measures, but after filtering, the category is dominated by AEP based measures (6 out of 8 ) such as the middle latency auditory-evoked potential index (MLAEPi; Tsurukiri et al., 2013). Two remaining measures, based on motor and somatosensory evoked potentials, were both the least consistent measures after the filtering process $(C I<0.44$ at $\mathrm{S} 1.6)$.

Given the high consistency ( $C I=0.80$ at S1.6, up 33\% from S1.2) both within and across frequency bands, the category seems to indicate that overall neural responsiveness to especially auditory responses seems to be reduced during anesthesia, DOC, and sleep. However, as ERP based measures quantify how the brain responds to information or perturbation, they are subject to modulation by anything that reduces processing in the relevant domain. While findings in studies on perceptual thresholds, for example, show that specific ERP components to auditory or visual stimuli can indicate whether the stimuli were apparent to conscious perception or not (Railo et al., 2011), the same components vanish for an otherwise strong stimulus when subjects attend to another modality (Nilsen et al., 2019). Therefore, whereas observations of sensory ERPs may indicate that a subject is conscious, an 
absence of sensory ERPs does not necessarily indicate unconsciousness, simply because consciousness per se is not sufficient for perceiving a specific stimulus, whereas perception of a specific stimulus necessitates consciousness.

Taken together, ERP as a category is consistent across measures, conditions, and frequencies, and consists of several reproduced and consistent individual parameters. The category also has appeal because of its perturbational nature (i.e. probing deterministic, causal properties of the system) and its reliance on conscious perception of sensory stimuli.

\subsubsection{Signal diversity}

The category signal diversity includes measures that quantify the functional differentiation or the spatiotemporal variability of either spontaneous (e.g. Lempel Ziv complexity; LZc; Schartner et al., 2015) or evoked (e.g. PCI; Casali et al., 2013) EEG recordings. The signal diversity category has the largest number of unique measures after the filtering process $\left(N_{\mathrm{M}}=\right.$ $15)$ and is the most consistent category $(C I=0.75$ at $\mathrm{S} 1.6)$ besides ERP. Furthermore, signal diversity measures are relatively independent of which frequency bands are in focus $(C I>$ 0.74 for all bands). This suggests that temporospatial functional differentiation is generally reduced in most or all states of unresponsiveness, with DOC patients showing the least consistent decrease $(C I=0.57)$ due to a relatively large fraction of non-significant findings (41.86\%). This may reflect the difficulty of accurately diagnosing DOC in patients (Schnakers et al., 2009).

One reason why signal diversity has become a popular basis for measures of consciousness is that prominent theories propose that the physical substrate of consciousness has to function as an integrated whole while simultaneously having the capacity for differentiated information processing (Sergent \& Dehaene, 2004; Tononi, 2004). However, signal diversity measures 
applied to spontaneous EEG data are generally unable to separate information integration and differentiation, because complexity can be confounded by noise (but see Pincus, 1991). In contrast, the perturbation-based signal diversity measure PCI, which is an index that quantifies the complexity of averaged TMS-evoked EEG signals, aims to capture spatiotemporal differentiation of the "deterministic", "causal" parts of the recorded responses (Massimini et al., 2009).

Taken together, signal diversity continues to be a promising category of EEG-based measures of consciousness. Especially considering that spontaneous EEG is fast and easy to employ and the required algorithms for calculating signal diversity are well studied and based on relatively rigorous definitions from information theory, physics, and mathematics. However, newer results have shown that signal diversity might be regionally differentiated and that global measures may not be sufficiently specific (Schartner, Pigorini, et al., 2017). For example, when measuring EEG with eyes closed, synchronous alpha-activity in occipital regions may lead to a drop in the global measures of signal diversity even without an overall change in state of consciousness (Farnes et al., 2020).

\subsubsection{Time-frequency}

The time-frequency category includes EEG measures that are based on time-frequency decompositions of either spontaneous signals (e.g. Median Spectral Frequency; MSF; Jordan et al., 2007) or evoked potentials (TEP-frequency power; Sanders et al., 2018). Time-frequency is the category with the highest amount of unique studies both before and after the filtering process $\left(N_{\mathrm{S}}=129\right.$ and $N_{\mathrm{S}}=111$, respectively), but is among the least consistent $(C I=0.14$ at $\mathrm{S} 1.6)$. However, while the low consistency is generally across conditions, it also has a high degree of variance within specific frequency bands after the 
filtering process, ranging from $\mathbf{b b C I}=0.70$ to $\boldsymbol{\alpha} C I=0.22$. The overall low consistency of the time-frequency category is highly influenced by two major contributors; BIS with a highly consistent decrease during states of unresponsiveness $(C I=0.95$ at $\mathrm{S} 1.6)$, and power in the delta band showing a strong increase $(C I=0.74$ at $\mathrm{S} 1.6)$. Both measures have over 40 results registered and account for $\sim 38 \%$ of all the results in the time-frequency category.

Time-frequency analysis is generally observational in nature and changes in the power frequency spectrum are a common observation in many different conditions of unresponsiveness (Finke et al., 2018; Northoff, 2017; Pagel, 2012). However, while time-frequency analysis has a strong tradition within studies of sleep, anesthesia, and DOC, there is a wide variety in the particular aspects being quantified. Some examples include measures of the spectral edge frequency that quantify the skew in the frequency power distribution (Jordan et al., 2007), power spectral density investigating power in a specific band of frequencies, and BIS capturing several aspects at once.

Taken together, however, while single parameters may be good classifiers of conscious state, the time-frequency category as a whole lacks a consistent interpretation for why one should expect specific effects, although several lines of research suggest that these changes can be attributed to changes in baseline operating modes such as information processing or cycling between functional task or rest states (e.g. Sanchez-Vives et al., 2017; Steriade et al., 1990).

\subsubsection{Multi-parameter / Other}

EEG measures that are based on the combination of multiple EEG measures from different categories are allocated to the multi-parameter category in this review (e.g. Cerebral State Index; Pilge et al., 2011, or Multivariate Model Analysis; Engemann et al., 2018). In the 
"Final Table" (Table 1, S1.6), none of the multi-parameter based measures survived the filtering process due to low reproduction rate.

For the category other, only Microstates remained, out of four, with eight studies out of 11. Microstates characterize temporal properties (here we summarized average duration) of four unique motifs in the EEG topography (Wenzel et al., 2019). While this parameter was fairly consistent within frequency bands $(C I=0.80$ for broadband, delta, theta, and gamma; $C I=$ 0.59 for alpha and beta), this was based on a relatively small number of results, five and nine, respectively. Taken together, we cannot really conclude much about these categories given the literature covered. However, microstate analysis may be a promising avenue for further research, especially in anesthesia.

\subsubsection{Summary}

The different categories vary in how affected they were by the filtering process and in their consistency over conditions, individual parameters, and frequency bands. However, the categories signal diversity and ERP are highly robust to our filtering steps and typically decrease from awake to all conditions of unresponsiveness yielding a high $C I$ value. This suggests that both categories of measures capture commonalities between conditions. All the other categories defined in this review are either lacking in reproduction or are non-informative due to their inconsistency in the effect direction. It could be the case that categories as such might not be the right level of conceptualization of EEG measures of consciousness, due to certain categories being more dependent on the exact implementation of parameters such as time-frequency and connectivity. Thus, the findings on the category level should be interpreted with caution and in light of relevant context. 


\subsection{Results for individual measures}

This section focuses on individual EEG measures. To get a more conclusive overview of EEG measures, we performed several exclusion steps (see Figure 1, and Suppl. 1). This resulted in a "Final Table" consisting of the 48 remaining EEG measures that were generally robust against the exclusion criteria used in this review. Of these 48 measures, 21 measures have at least 10 results (Table 3). The complete tables, including all measures investigated from S1.0 to S1.6, are shown in the supplementary data sheet (SDAT: Supplementary_Measures). 


\section{[TABLE 3]}

\subsubsection{Results for individual measures}

Among the 21 remaining measures (Table 3), seven are signal diversity measures, seven are time-frequency measures, four are ERP measures, and three are connectivity measures. Of the 21 measures, 13 of these had been studied in all three conditions (anesthesia, DOC, sleep). Of these 13 , nine measures are relatively consistent across conditions $(C I>0.5)$. Of these nine measures, two are time-frequency measures, five are signal diversity measures, and two are ERP measures. Most $\left(N_{\mathrm{M}}=7\right)$ of these nine relatively consistent and generally reproduced measures are calculated on a broadband or a multi-band frequency range, while the remaining two measures are limited to either the delta band or the delta-beta range.

Finally, we selected the six most consistent measures $(C I>0.8)$ for further discussion. Of these six measures, four are signal diversity measures, five are broadband or multi-band-based, and two have technical or practical comments associated with them (see Suppl. 1, S1.6). However, importantly, these comments were not explicitly searched for, and should thus only be regarded as informing discussion and possibly point towards future studies that could be performed to validate what a measure captures.

\subsubsection{Spectral Entropy (SpEn)}

Spectral entropy (SpEn; as used in e.g. Kaskinoro et al., 2011; Kim et al., 2012), calculated on a broadband frequency range, is one of the two most consistent measures (tied with LZc) that was reproduced across all major conditions $(C I=0.89)$. SpEn decreased, compared to wakefulness, in all conditions except ketamine anesthesia and coma for which there were no 
results, and one non-significant result in REM sleep (out of one). SpEn was mostly investigated during general anesthesia $(\sim 78 \%)$.

SpEn is a commercially available anesthesia monitor (Viertio-Oja et al., 2004) which estimates the uniformity of the power spectral distribution of the spontaneous EEG by calculating the entropy of the distribution, and is thus a practical measure in many situations. Specifically, SpEn combines the so-called state entropy and response entropy measures, which are calculated by taking the Shannon entropy of the normalized Fourier transformed signal for specific frequency ranges $(0.8-32 \mathrm{~Hz}$ and $0.8-47 \mathrm{~Hz}$, respectively). While the individual components, state entropy, and response entropy were excluded because they were not replicated in DOC or sleep, they might arguably be merged with spectral entropy, though the overall consistency would have been similar, both for the signal diversity category and for the specific measure SpEn as well (see SDAT: Supplementary_Measures). However, there are also indications that $\mathrm{SpEn}$ is influenced by electrical signals generated by muscle activity (Viertio-Oja et al., 2004), thus, state entropy, which is calculated on frequencies below where EMG is thought to occur, could be a "cleaner" measure of unresponsiveness, especially when muscle relaxants are not used.

\subsubsection{Lempel-Ziv complexity $(\mathrm{LZc})$}

Lempel-Ziv complexity (LZc; as used in e.g. Farnes et al., 2020; Schartner et al., 2015), calculated on a broadband frequency range, is equal to $\mathrm{SpEn}$ in terms of consistency $(C I=$ 0.89). LZc decreased, compared to wakefulness, in all conditions except coma for which there were no results, and one non-significant result in propofol anesthesia (out of six). Like most measures in the "Final Table" with at least 10 results (Table 3), LZc was mostly investigated during general anesthesia ( $65 \%)$. 
LZc is an estimate of Kolmogorov complexity (i.e. descriptive complexity) and calculates the compressibility of the binarized analytical signal. The more periodic or ordered the analytic EEG signal is across channels and time, the lower the LZc value will be. Thus, LZc, like other measures of signal diversity, aims to capture differentiation in the signal. It can be calculated on almost every EEG signal and does not strictly rely on additional stimulation or brain recordings (e.g. TMS or MRI), which makes it practically applicable in many situations. However, like other complexity and entropy-based measures, LZc can confound complexity with noise in spontaneous EEG activity. To get a more direct estimate of the complexity of the underlying brain processes, and simultaneously reduce the confounding effects of noise, one can compute the LZc based on data resulting from sensory stimulation or direct perturbation (e.g. see PCI; Casali et al., 2013; Massimini et al., 2009). Further, the precise LZc values are affected by the specific implementation, such as the number of samples in an epoch, sampling rate, filters, number of channels, and normalization. Therefore, care should be taken when comparing absolute values of LZc between studies.

\subsubsection{Spectral edge frequency $95 \%$ (SEF95)}

Spectral edge frequency at the 95th percentile of the power spectral density (SEF95; as used in e.g. Jordan et al., 2007; Nieuwenhuijs et al., 2002), calculated on a broadband frequency range, is one of the two second most consistent measures (tied with ApEn) that was reproduced across all major conditions $(C I=0.83)$. SEF95 decreased, compared to wakefulness, in all conditions except ketamine anesthesia for which there were no results, one non-significant result in REM sleep (out of one), and two non-significant results (out of nine) in the condition "mix/other" anesthesia. SEF95 was mostly investigated in general anesthesia $(\sim 65 \%)$ 
SEF95 is another popular anesthesia monitoring tool and returns the frequency at which the area under the curve reaches a certain threshold of the total power, commonly $95 \%$. Thus, SEF95 captures how skewed the power spectral density distribution is towards the lower frequency ranges, a shift that is often associated with states of unresponsiveness as captured by other measures (Sanchez-Vives et al., 2017). SEF95 is commonly applied to spontaneous data, which together with fast and straightforward calculation, makes it applicable to most situations. However, as is the case for other related measures (e.g. spectral exponent; Colombo et al., 2019), it is also potentially vulnerable to contamination by electrical signals generated by muscle activity, which may increase power in high frequencies, thus shifting the spectral edge.

\subsubsection{Approximate entropy (ApEn)}

Approximate entropy (ApEn; as used in e.g. Fan et al., 2011; Ferenets et al., 2006), calculated on a broadband frequency range, is equal to SEF95 in terms of consistency $(C I=0.83)$. ApEn decreased, compared to wakefulness, in all conditions except ketamine anesthesia and coma for which there were no results, and one non-significant result (out of two) in both unresponsive wakefulness syndrome (UWS; formerly known as vegetative state) and MCS conditions. ApEn was mostly investigated in general anesthesia ( $65 \%)$.

ApEn captures the irregularity of the data based on the probability that similar patterns in the data do not repeat. It also has the same drawbacks as other signal diversity measures; e.g. dependence on the number of samples, preprocessing, and exact implementation. While these drawbacks create problems for generating absolute values for comparison of ApEn between studies (e.g. creation of general classification thresholds), they do not create major concerns within a study as long as the measure is calculated using the same parameters for all 
conditions. A strength of ApEn compared to LZc and SpEn, is that it can in principle distinguish noisy from chaotic time series in certain conditions (Pincus, 1991), however, given the overall high consistency for signal diversity measures (see Figure 2), it is unclear whether EEG signals naturally visit these conditions.

\subsubsection{Auditory evoked potential at $100 \mathrm{~ms}$ (AEP N100)}

The characteristics of the auditory evoked potential at $~ 100 \mathrm{~ms}$ (AEP N100; as used in e.g. Haenggi et al., 2009; Tavakoli et al., 2018), calculated on the delta-beta frequency range, is the third most consistent measure (tied with PeEn) that was reproduced across all major conditions $(C I=0.81)$. AEP N100 decreased, compared to wakefulness, in all conditions except for sevoflurane, ketamine, and "mix/other" anesthesia for which there were no results, and one non-significant result in NREM sleep (out of two). Unlike SpEn, LZc, SEF95, PeEn, and ApEn, AEP N100 was mostly investigated in DOC (40\%) but was among the least studied measures of the "Final Table" with a total of 10 results.

EEG recorded ERPs are often used in the clinic and in basic research. N100 is an ERP component that occurs as a negative peak in the signal roughly $100 \mathrm{~ms}$ after a sensory stimulus. It can be seen in trial-averaged data and is associated with perception of sensory input (e.g. Yurgil \& Golob, 2010). The N100 component is usually regional, with most studies focusing on primary sensory areas, secondary associative areas, or prefrontal areas, with the latter being more associated with predictability and relevance of the stimuli (Schwartze et al., 2013). When analyzing ERPs, the peak amplitude, latency, or frequency content of a particular component is typically used. It is relatively straightforward, and ERP-based measures are easily applicable to everyone, only requiring a stimulus such as audibly repeating and deviating tones (i.e. oddball task). However, most ERP measures require a 
fairly large number of repetitions ( $>100$ trials) to achieve a sufficiently high signal-to-noise ratio (SNR), and are dependent on both intact pathways for sensory processing and attention to the signal (King et al., 2013). Further, because the N100 requires multiple repetitions for obtaining a good SNR, several studies performed the analysis at the group level (De Salvo, 2015; Yppärilä et al., 2004), and it is far from clear how reliable the N100 is at the individual level, since $24 \%$ of coma patients also showed an N100 response different from baseline in one study (Daltrozzo et al., 2009).

\subsubsection{Permutation entropy (PeEn)}

Permutation entropy (PeEn; as used in e.g. Shalbaf et al., 2013; Stefan et al., 2018), calculated on a broadband frequency range or on any individual band (delta, theta, ...), is equal to AEP N100 in terms of consistency $(C I=0.81)$. PeEn decreased, compared to wakefulness, in all conditions except for ketamine anesthesia and coma for which there were no results, two non-significant results (out of six) for both MCS and UWS conditions, and one non-significant result (out of three) for the "mix/other" anesthesia condition. PeEn was mostly investigated in general anesthesia $(\sim 47 \%)$.

PeEn estimates the randomness of the data by calculating the entropy of the distribution of ranked ordinal amplitude patterns. In this sense, it is similar to ACE (Schartner et al., 2015) in that it first transforms the data into specific patterns (coalitions), and then calculates the evenness of the distribution of the occurrences of these patterns. However, in contrast to ACE, the "coalitions" used in permutation entropy are ordered in time (within channels) rather than in space (between channels). Thus, it is a measure that captures the entropy of temporal patterns of activity in time series. In general, PeEn faces similar issues as all other signal diversity measures, but it has only one free parameter that needs to be set a priori, unlike 
ApEn, which has two, and LZc and SpEn, which has zero. However, while PeEn is overall consistent in its effect direction, it was not able to discriminate between patients in general anesthesia who were able to respond during an isolated forearm technique (IFT) paradigm and those who could not (Gaskell et al., 2017).

\subsubsection{Honourable mentions}

Two measures had a higher consistency than those mentioned above; Bispectral index (BIS) and the combination (MLAEPi \& AAI) of Middle latency auditory evoked potential index (MLAEPi) and A-line autoregressive index (AAI), both with $C I=0.95$. These were not included in the discussion above because they have not been reproduced across all three major conditions (anesthesia, DOC, sleep). Thus, we found no study where BIS has been used in DOC patients, while for MLAEPi \& AAI we found no use of it in sleep. Possibly, such studies may have failed to be discovered by our search or may have been excluded because they lacked an explicit wake control. However, using a free literature search, we found studies investigating both measures in the non-reproduced conditions. For example, the prognostic and diagnostic value of BIS has been studied in DOC patients (Fàbregas et al., 2004; Schnakers et al., 2008; Shibata et al., 2005), and MLAEPi\&AAI has been tested in sleep (Deiber et al., 1989).

BIS is a single-valued weighted sum of several spectral and bicoherence parameters, and is the most studied measure included in this review due to its prominence as an anesthesia monitor and reference measure when testing other measures of consciousness. However, the algorithm underlying BIS is proprietary and not open to researchers for detailed investigation. While BIS is a very consistent and highly reproduced measure, there are limitations that warrant mention. For example, BIS is noted to not correlate well with awareness during 
general anesthesia (Avidan et al., 2008), and can be affected and possibly confounded by electrical signals generated by muscle activity, and therefore by neuromuscular blockers (Choi et al., 2017; Fuentes et al., 2008; Lu et al., 2008). BIS is also affected by age (Lysakowski et al., 2009), is unreliable in intellectually disabled children (Valkenburg et al., 2009), has high inter-subject variability (Kaskinoro et al., 2011), and does not separate between IFT responders and non-responders (Gaskell et al., 2017).

MLAEPi is calculated on the 10-75 ms auditory ERP response evoked by a series of clicks and is a part of several commercial anesthesia monitors. One such monitor is the AAI, which performs an adaptive and automatic extraction of MLAEPi. The MLAEPi measure targets the early response typically associated with thalamic and primary sensory cortical processing of an auditory stimulus, and is less dependent on attentional resources being directed to the stimulus than the later responses $(>100 \mathrm{~ms})$ (Railo et al., 2011). While MLAEPi is normally reduced during general anesthesia, it has also been found to be reduced according to dosage of the opioid analgesic drug remifentanil independently of the sedation level (Supp et al., 2018). In addition, MLAEPi has been observed to be affected by analgesia while patients are conscious and responsive (Lu et al., 2010), and reduced by neuromuscular blockers (Lu et al., 2008).

The remaining 13 EEG measures of Table 3 not discussed above are not described in detail here, although the measures were robust against the filtering process, and most $\left(N_{\mathrm{M}}=8\right)$ of them have a relatively consistent effect direction $(C I>0.50)$. The 27 remaining measures of the "Final Table" (see SDAT: Supplementary_Measures at S1.6) that were excluded because we found less than 10 test results, require further studies, including both reproduction of existing results and/or extension to other conditions of unresponsiveness. 


\subsubsection{Summary}

Altogether, while the final EEG measure table (Table 3) shows that the search for a generally consistent and reproducible EEG measure of consciousness is far from over, the eight EEG measures discussed here seem to be promising in terms of consistency and reproduction across conditions. The measures LZc and SEF95 stand out because they have been studied in all specific conditions except one (coma and ketamine anesthesia, respectively), are highly consistent across conditions $(C I>0.83)$, highly reproduced (results $>16)$, had no technical concerns associated with them, are applicable to spontaneous EEG with no extra equipment, and can be automatically calculated in real time by open source algorithms. In addition, SpEn stands out in terms of being straightforward to implement, as it is relatively independent of exact implementation, although it can be affected by electrical signals generated by muscle activity.

\section{DISCUSSION}

This review is intended to provide a comprehensive overview of proposed EEG-based measures of consciousness. To our knowledge, such an overview has been missing so far. However, given that most studies reviewed here did not record subjective reports upon awakening (if at all), this review covers how various measures generally behave during conditions of unresponsiveness.

We find that measures of the two categories signal diversity and ERP, consistently decrease during unresponsiveness relative to normal wakefulness. This result is based on several studies across multiple conditions and implementations. Furthermore, measures of both categories are relatively independent of specific frequency ranges of the underlying EEG signal and instead are commonly calculated on a broadband frequency range. The remaining 
categories time-frequency, connectivity, and other were relatively inconsistent in their effect directionality.

Among the single measures, nine out of 255 measures survived the filtering process, were reproduced across all major conditions (anesthesia, DOC, sleep), were based on at least 10 results, and were relatively consistent $(C I>0.5)$. Of these nine measures, five were signal diversity measures, two were time-frequency measures, and two were ERP measures. Finally, seven out of these nine measures were calculated on a broadband frequency range. Among the time-frequency measures, SEF95 stands out as being both highly consistent among conditions and widely reproduced. Although BIS was one of the two most consistent measures overall (tied with MLAEPi\&AAI), it lacked results in REM and NREM sleep. Among the ERP measures, auditory ERPs seem to give the most consistent results in general, with AEP N100 being the most consistent and overall reproduced ERP measure besides MLAEPi\&AAI. However, MLAEPi\&AAI lacked results in DOC. Finally, among the signal diversity measures, there are several good candidates to choose from, but care has to be taken in terms of preprocessing, exact implementation, and parameter settings. SpEn is unique in this regard, as it is quite straightforward to implement. While it can be affected by electrical signals generated by muscle activity (Kim et al., 2012), this can be counteracted in part by using only the state entropy component, which only considers low frequencies $(<32 \mathrm{~Hz})$ in the power spectra, thus excluding the dominant muscle activity frequency range.

In terms of categories, our results suggest that the complexity of functional dynamics, as measured by signal diversity measures, and cortical responsiveness to external stimuli, as measured by ERP measures, are two general properties that seem promising for future development of measures of states of consciousness. The perturbational complexity index 
(PCI; Casali et al., 2013) is interesting in this regard as it combines both aspects by capturing the signal diversity of TMS-induced ERPs, though it does not show a clear effect direction for ketamine anesthesia and REM sleep. While the lack of a clear effect direction in ketamine anesthesia and REM sleep, relative to wakefulness, has been proposed to be due to vivid dreams in these conditions (Sarasso et al., 2015), evidence for dreams have also been found in both NREM sleep and propofol anesthesia when participants have been asked to report their subjective experiences immediately upon awakening, before they are forgotten (Leslie et al., 2007, 2009; Nielsen, 2000; Noreika et al., 2011a).

The relatively high degree of inconsistency among time-frequency and connectivity measures might be due to their dependence on the cortical region of interest and directionality of connectivity, respectively. For example, it is currently debated whether the front or the back of the brain is more involved in conscious processing (Boly et al., 2017; Odegaard et al., 2017), and many observations throughout the past few decades have indicated that changes in information flow during states of unresponsiveness are direction-specific (e.g. Lee et al., 2009; Maksimow et al., 2014). Therefore, a more detailed approach might be more informative for future reviews of connectivity based measures of consciousness (but see section 4.1.3).

Given that statistically significant findings are more prone to be published (for a comparison between traditional and pre-registered studies, see (Scheel et al., 2020), care should be taken when interpreting the performance of both categories and individual measures. However, our consistency index represents an attempt to partly compensate for this by weighing opposite effect directions more heavily than non-significant results. Furthermore, signal diversity and 
$E R P$ are again the most promising categories as they showed relatively low percentage of non-significant results: $\sim 24 \%$ and $\sim 8 \%$, respectively.

While measures in the categories ERP, time-frequency, and signal diversity can be interpreted on a linear scale (e.g. amplitude of the N100, signal entropy, power), some others, especially multi-parameter and some connectivity measures, show diverse non-uniform alterations between states (e.g. regional or direction specific changes). Often, these changes are analyzed by a machine learning classifier and only the overall accuracy of classification is reported (e.g. Lee et al., 2009). Studies that only reported accuracy of a classifier were not included in our review since they are difficult to interpret.

Many of the measures in the "Final Table" have comments associated with them. These comments point out specific cases where the measure is less reliable or valid. For example, BIS is unable to differentiate between subjects in anesthesia who can and cannot respond during the isolated forearm technique (Gaskell et al., 2017), mutual information is in some cases not showing a return to pre-anesthesia levels following return of consciousness (Liang et al., 2015), and the spectral power of the TMS evoked potential does not discriminate between reports and non-reports of dream content following rapid awakening from deep sleep (Lee et al., 2019). We did not explicitly search for studies performing such contrasts, noting only those who showed up during the literature review. However, it should be a requirement for a valid measure of phenomenological consciousness that it can capture obvious cases of subjective experience such as return to responsiveness following anesthesia. Furthermore, some measures seem to be affected by electrical activity generated by muscle activity (e.g. BIS; Schuller et al., 2015) or are unreliable for certain demographics and patient groups (e.g. delta power increases also in locked-in-syndrome patients; Babiloni et al., 2010). While such 
comments are not necessarily detrimental for a measure, they do indicate that the measure might be capturing something else than EEG activity directly associated with consciousness, or that it is unreliably in the presence of certain noise factors. For example, muscle activity is often inhibited by neuromuscular blockade in anesthesia and muscle tonus is reduced during sleep. Thus, because electrical activity generated by muscle activity is typically mixed in with neural signals in EEG recordings, overall muscle tonus could be a confounding factor for EEG-based measures of consciousness. Future studies should not only seek to reproduce existing measures, but test them against specific protocols such as intermittent awakening from sleep and anesthesia, isolated forearm technique, muscle relaxants, and other edge cases. Specifically, using within-state paradigms to study how measures change between situations with and without report of conscious experience when the physiological state as a whole is comparable, may be useful for understanding which measures are driven by changes relevant to consciousness and which depend on confounding differences between states.

Finally, both ERP and signal diversity measures have been shown to be influenced by processes that are unlikely to relate to the presence of consciousness per se. For example, the auditory evoked $\mathrm{P} 300 \mathrm{~b}$ response has been shown to disappear if the subject is paying attention to something else than the auditory stimulus (Bekinschtein et al., 2009), and the signal diversity based measure LZc has been shown to be reduced when subjects close their eyes (Farnes et al., 2020) or increase during psychedelic experiences (Schartner, Carhart-Harris, et al., 2017). In other words, certain non-rest cognitive or physiological conditions in which subjects are conscious could serve as a test against false negatives. Testing against such cases is also of clinical importance since for example closed eyes and inattentiveness could reasonably occur during conditions like sleep and anesthesia, without altering the underlying 
state of consciousness (i.e. subjects could be dreaming). Thus, future studies should also test measures against such modulations (King et al., 2013).

\subsection{Limitations and considerations}

\subsubsection{Consciousness $\neq$ unresponsiveness}

One of the biggest limitations is that the review is not investigating EEG measures of consciousness directly. This is due to the fact that it has not been standard for the subjective experience of participants to be recorded in most studies of consciousness (e.g. by waking the participants up during sedation or sleep and explicitly asking for their subjective experience prior to being awakened). In fact, studies where researchers do control for subjective experience are extremely rare and a systematic review of EEG measures of consciousness is not possible, yet. This is despite the growing knowledge about the presence of subjective experience in several states of unresponsiveness. For example, by use of intermittent awakenings dream reports have been recorded in up to $75 \%$ of awakenings in NREM sleep versus up to $93 \%$ in REM sleep (Nielsen, 2000). Further, healthy participants under the effect of general anesthetics report dreams upon awakening almost $60 \%$ of the time (Noreika et al., 2011b), and EEG-based communication has been established with patients in UWS (e.g. Cruse et al., 2011; Owen et al., 2006). Additionally, while subjective experiences have been reported to occur in anesthesia, sleep, and DOC, some argue that ketamine anesthesia and REM sleep are special in this regard as the subjective experiences are reported to be more vivid and complex than in other conditions such as propofol anesthesia and NREM sleep (Casarotto et al., 2016). Together, this suggests that the commonly used operational definition of consciousness as closely related to the capacity to respond reliably to stimuli, i.e. responsiveness, is insufficient in terms of testing measures of consciousness. For the current 
review, this limitation means that the consistency of a measure or category might not be a valid estimate of "quality" because if there is heterogeneity in the underlying state of consciousness, then a highly consistent effect direction is not necessarily a desirable feature of a measure of consciousness. To account for this, future studies should take care to consider subjective reports immediately after test subjects reemerge from unresponsive states, if they wish to make claims about changes in consciousness (and capacity for objective measures to track it). However, subjective reports are vulnerable to several confounds such as confabulation, no or false memories, or misunderstanding (see Schwitzgebel, 2008) for a discussion).

\subsubsection{Consistency}

We might do injustice to specific measures or categories by ranking them in terms of consistency. After all, the different conditions included here (anesthesia, sleep, DOC) vary wildly in terms of the mechanisms underlying them. Furthermore, the presence or absence of consciousness is likely variable in different sub-conditions (e.g. REM vs NREM), which may make the summary tables misleading. As such, we might give the impression that a measure or category of measures is not promising if one only judges them based on these coarse summaries. Therefore, we emphasize the importance of looking at the full tables (see supplementary datasheets) and judging measures based on the field's evolving knowledge regarding which conditions are permissive of conscious experience and which are less so. Still, we believe that a general measure of consciousness should be independent of the physiological, psychological, or pharmacological state of a subject, as long as it reliably captures variations in consciousness. Importantly, as we learn more about the state of consciousness in specific conditions, a consistency index might prove invaluable in terms of 
ruling out measures that treat a state of consciousness as a state of unconsciousness. This means that the $C I$ metric used here to provide a summary of the results for each measure and category might or might not be useful depending on future knowledge of the state of consciousness of various conditions. Further, given a heterogeneity in technical implementation, measurement characteristics, and other methodological differences between studies employing the same measure, $C I$ might be informative in terms of how much such variation matters for a particular measure. A high $C I$ could also indicate that a measure is sensitive to something else that normally correlates with conscious or responsive state, such as arousal, wakefulness, muscle tension, or outwards attention. In theory, several EEG measures should be sensitive to muscle tonus and therefore change naturally with paralysis of the body (as is common in general anesthesia or REM sleep). Therefore, additional recordings of electrical signals generated by muscle activity would be beneficial to test to what degree measures of consciousness reflect changes in muscle tonus rather than consciousness.

\subsubsection{Methodological limitations}

In terms of methodological limitations, this review is only based on the results of each paper and does not evaluate the quality of the studies, nor technical or methodological specifics beyond those stated. However, measuring the quality of the literature would be based on subjective evaluation due to the many different methodologies, conditions, and research questions employed in the studies. This could lead to not only difficulty in replicating the review itself, but also to misunderstandings of nuances related to each measure. Further, evaluating technical and methodological details comes with the risk of increased granularity and issues in how to interpret these differences. Given the huge variability in the studies covered here, a comparison of for example the influence of number of electrodes or reference 
placement, needs to be targeted by specific reviews. To facilitate comparison, future research should strive to use open source toolboxes in analysis and/or share their code, use standardized EEG setups and preprocessing steps, and as far as possible, document experimental or clinical conditions.

It is also debatable whether our filtering process is a valid or optimal approach to narrow down the literature. However, we carefully defined and considered each single step in the most objective way we could, and every EEG measure is treated exactly the same independent of the article's journal, authors, writing quality, or interpretation. The objective treatment of each study is an advantage of this review because the degree of reproduction, as captured by consistency of the effect direction, is generalized over variation in exact implementation such as preprocessing steps and choice of parameters. Furthermore, it allows for future extension of the results presented here.

Restricting the initial literature search to only the past 10 years comes with the limitation that EEG measures which have not been used in the past 10 years are not part of this review. However, if a measure has not been used in the past 10 years we assume it is because that measure is not promising, practical, or that it has been replaced by better alternatives. While the extended literature search, applied for each EEG measure "Y", may have included papers that were out of the scope of the first search, any new measure " $X$ " investigated in conjunction with measure "Y" was not added to the table. From our point of view, this approach was necessary to treat every measure the same way and to reduce the complexity of the review.

Finally, certain measures were merged that perhaps should not have been (e.g. MLAEPi and AAI), while other measures should perhaps have been merged, but were not (e.g. state and 
response entropy). Similarly, we decided to split certain measures according to which frequency band was focused on, due to a large literature indicating that the power spectrum skews towards lower frequencies in most of the conditions investigated (Koch et al., 2016), and that frequency bands are indicative of different cognitive and physiological processes (Herrmann et al., 2016; Klimesch, 1999, but see Başar et al., 1999). Thus, it could be argued that a measure such as directed weighted phase lag index (dwPLI) calculated in the delta band is a different measure than dwPLI calculated on the alpha band (opposite effect directions, see SDAT: Supplementary Measures). Additionally, splitting based on frequency bands is common across all categories employed in this review. For certain measures we could have split also on region (e.g. anterior, posterior, global), connectivity directionality (e.g. front-to-back, back-to-front), and implementation (e.g. relative vs. absolute power, MLAEPi vs. AAI). While increased granularity could reduce inconsistencies, a lack of merging could cause removal of measures due to a lack of reproduction. However, exactly how to merge or split measures is debatable. As such, we opted for only one level of distinction. Thus, the estimated consistency of a category might be interpreted more as a reflection of the construct reliability of a category, rather than construct validity. Another limitation caused by merging or splitting is the observations regarding specific frequency bands. As we merged with respect to consistency, this resulted in the consistency of specific frequency bands to be extrapolated (see Suppl. 4). While this caused in most cases only a minor deviance in overall consistency (less so for more reproduced categories, see SDAT: Supplementary Categories), care should be taken when interpreting the frequency band specific findings.

\subsection{Future steps}


Future reviews and meta-studies should carefully consider how to merge similar measures, how to categorize them in a fruitful manner, and how to split up results that investigate the same measure in different ways (e.g. using different implementations or different parts of the signal). Furthermore, there are at least three areas that would be interesting to pursue to improve and expand on the results of this review: (1) cross condition reproduction and direct comparison of measures, especially in conditions with more wakelike EEG such as REM sleep and ketamine anesthesia which can cause issues for several popular tools such as BIS and MLAEPi (Hirota, 2006), (2) testing against possible confounds and conditions which could produce false negatives and positives such as muscle relaxants or diverted attention, and, (3) probe the underlying state of consciousness of the subjects using subjective reports through for example intermittent awakening or isolated forearm technique. In addition to further testing or introducing new measures, the field may benefit from converging on how to best classify the various unresponsive conditions with regards to their assumed state of consciousness. This last point is essential if we wish to find a general marker for consciousness per se, rather than a marker that merely reflects our preconceived notions regarding which conditions are (and are not) associated with consciousness.

\section{CONCLUSIONS}

Overall, the quest for a robust and consistent EEG-based measure of consciousness is difficult to fulfill and there still seems to be a long way to go before a satisfactory measure is obtained.

The two categories of measures, signal diversity and ERP, are fairly informative, robust, and consistent in their effect directions. This suggests that measures based on signal diversity, capturing functional differentiation or "predictability" of the signal, and measures based on ERPs, capturing functional responsiveness to external stimuli, might be fruitful properties to 
investigate further. Among the individual measures, spectral entropy (SpEn), Lempel Ziv complexity (LZc), and spectral edge frequency 95\% (SEF95) stand out in terms of consistency, reproducibility, and practicality. However, several candidate measures have not been sufficiently reproduced within or between conditions, thus more research is needed in order to properly compare proposed measures in the future.

Nevertheless, it is important to keep in mind that for all EEG-based measures covered by this review, it is far from clear if they truly reflect consciousness (i.e. phenomenal, subjective experience; see Box 1), since the state of consciousness of each subject was in most cases inferred from the level of behavioral responsiveness. Thus, to make real progress towards finding a reliable measure of consciousness, we argue that it is essential to collect detailed reports from participants about their subjective experiences as soon as possible after emerging from unresponsiveness. If immediate or delayed reports are unavailable or unreliable, we advise researchers to remain agnostic about the presence or absence of consciousness. We propose that this should be the new standard for this field of research.

\section{Acknowledgments}

We thank Peder Isager and Andrew Lee for feedback on an earlier version of this manuscript.

\section{Funding}

Supported by the European Union's Horizon 2020 Framework Programme for Research and Innovation under the Specific Grant Agreements No. 785907 (Human Brain Project SGA2), WAP also by No. 945539 (Human Brain Project SGA3), the Norwegian Research Council FRIMEDBIO (Grant No. 262950), and a UiO Convergence Environment Program project: Conscious Brain Concepts. 


\section{Disclosure}

The authors report no conflict of interest. 


\section{Bibliography}

Albright, T. D., Jessell, T. M., Kandel, E. R., \& Posner, M. I. (2000). Neural science: a century of progress and the mysteries that remain. Neuron, 25 Suppl, S1-S55. https://doi.org/10.1016/S0092-8674(00)00251-8

Avidan, M. S., Zhang, L., Burnside, B. A., Finkel, K. J., Searleman, A. C., Selvidge, J. A., Saager, L., Turner, M. S., Rao, S., Bottros, M., Hantler, C., Jacobsohn, E., \& Evers, A. S. (2008). Anesthesia awareness and the bispectral index. The New England Journal of Medicine, 358(11), 1097-1108. https://doi.org/10.1056/NEJMoa0707361

Babiloni, C., Pistoia, F., Sarà, M., Vecchio, F., Buffo, P., Conson, M., Onorati, P., Albertini, G., \& Rossini, P. M. (2010). Resting state eyes-closed cortical rhythms in patients with locked-in-syndrome: An eeg study. Clinical Neurophysiology: Official Journal of the International Federation of Clinical Neurophysiology, 121(11), 1816-1824. https://doi.org/10.1016/j.clinph.2010.04.027

Başar, E., Başar-Eroğlu, C., Karakaş, S., \& Schürmann, M. (1999). Are cognitive processes manifested in event-related gamma, alpha, theta and delta oscillations in the EEG? Neuroscience Letters, 259(3), 165-168. https://doi.org/10.1016/s0304-3940(98)00934-3

Bekinschtein, T. A., Dehaene, S., Rohaut, B., Tadel, F., Cohen, L., \& Naccache, L. (2009). Neural signature of the conscious processing of auditory regularities. Proceedings of the National Academy of Sciences of the United States of America, 106(5), 1672-1677. https://doi.org/10.1073/pnas.0809667106

Bertini, M., Ferrara, M., De Gennaro, L., Curcio, G., Moroni, F., Babiloni, C., Infarinato, F., Rossini, P. M., \& PhD, F. V. (2009). Directional information flows between brain hemispheres across waking, non-REM and REM sleep states: An EEG study. Brain Research Bulletin, 78(6), 270-275. https://doi.org/10.1016/j.brainresbull.2008.12.006

Boly, M., Massimini, M., Tsuchiya, N., Postle, B. R., Koch, C., \& Tononi, G. (2017). Are the Neural Correlates of Consciousness in the Front or in the Back of the Cerebral Cortex? Clinical and Neuroimaging Evidence. The Journal of Neuroscience: The Official Journal of the Society for Neuroscience, 37(40), 9603-9613. https://doi.org/10.1523/JNEUROSCI.3218-16.2017

Boly, M., Seth, A. K., Wilke, M., Ingmundson, P., Baars, B., Laureys, S., Edelman, D. B., \& Tsuchiya, N. (2013). Consciousness in humans and non-human animals: recent advances and future directions. Frontiers 
in Psychology, 4, 625.

http://www.pubmedcentral.nih.gov/articlerender.fcgi?artid=3814086\&tool=pmcentrez\&rendertype=abstract Buzsáki, G., Anastassiou, C. A., \& Koch, C. (2012). The origin of extracellular fields and currents--EEG, ECoG, LFP and spikes. Nature Reviews. Neuroscience, 13(6), 407-420. https://doi.org/10.1038/nrn3241

Casali, A. G., Gosseries, O., Rosanova, M., Boly, M., Sarasso, S., Casali, K. R., Casarotto, S., Bruno, M.-A., Laureys, S., Tononi, G., \& Massimini, M. (2013). A theoretically based index of consciousness independent of sensory processing and behavior. Science Translational Medicine, 5(198), 198ra105. https://doi.org/10.1126/scitranslmed.3006294

Casarotto, S., Comanducci, A., Rosanova, M., Sarasso, S., Fecchio, M., Napolitani, M., Pigorini, A., G. Casali, A., Trimarchi, P. D., Boly, M., Gosseries, O., Bodart, O., Curto, F., Landi, C., Mariotti, M., Devalle, G., Laureys, S., Tononi, G., \& Massimini, M. (2016). Stratification of unresponsive patients by an independently validated index of brain complexity. Annals of Neurology, 80(5), 718-729. https://doi.org/10.1002/ana.24779

Chennu, S., Finoia, P., Kamau, E., Monti, M. M., Allanson, J., Pickard, J. D., Owen, A. M., \& Bekinschtein, T. A. (2013). Dissociable endogenous and exogenous attention in disorders of consciousness. NeuroImage: Clinical, 3, 450-461. https://doi.org/10.1016/j.nicl.2013.10.008

Chennu, S., O’Connor, S., Adapa, R., Menon, D. K., \& Bekinschtein, T. A. (2016). Brain Connectivity Dissociates Responsiveness from Drug Exposure during Propofol-Induced Transitions of Consciousness. PLoS Computational Biology, 12(1). https://doi.org/10.1371/journal.pcbi.1004669

Chernik, D. A., Gillings, D., Laine, H., Hendler, J., Silver, J. M., Davidson, A. B., Schwam, E. M., \& Siegel, J. L. (1990). Validity and reliability of the Observer's Assessment of Alertness/Sedation Scale: study with intravenous midazolam. Journal of Clinical Psychopharmacology, 10(4), 244-251. https://www.ncbi.nlm.nih.gov/pubmed/2286697

Choi, J. B., Na, S. H., Lee, S. Y., Kim, J. Y., Park, S. Y., Kim, J. E., Hong, S., An, J., Park, C. H., Kim, Y. C., \& Park, W. Y. (2017). Suxamethonium induces a prompt increase in the bispectral index. Medicine, 96(16), e6670. https://doi.org/10.1097/MD.0000000000006670

Colombo, M. A., Napolitani, M., Boly, M., Gosseries, O., Casarotto, S., Rosanova, M., Brichant, J.-F., Boveroux, P., Rex, S., Laureys, S., Massimini, M., Chieregato, A., \& Sarasso, S. (2019). The spectral exponent of the resting EEG indexes the presence of consciousness during unresponsiveness induced by 
propofol, xenon, and ketamine. NeuroImage, 189, 631-644.

https://doi.org/10.1016/j.neuroimage.2019.01.024

Crick, F., \& Koch, C. (1990). Towards a neurobiological theory of consciousness. Seminars in the Neurosciences, 2, 263-275. https://authors.library.caltech.edu/40352/

Cruse, D., Chennu, S., Chatelle, C., Bekinschtein, T. A., Fernández-Espejo, D., Pickard, J. D., Laureys, S., \& Owen, A. M. (2011). Bedside detection of awareness in the vegetative state: A cohort study. The Lancet, 378(9809), 2088-2094. https://doi.org/10.1016/S0140-6736(11)61224-5

Daltrozzo, J., Wioland, N., Mutschler, V., Lutun, P., Calon, B., Meyer, A., Pottecher, T., Lang, S., Jaeger, A., \& Kotchoubey, B. (2009). Cortical information processing in Coma. Cognitive and Behavioral Neurology: Official Journal of the Society for Behavioral and Cognitive Neurology, 22(1), 53-62. https://doi.org/10.1097/WNN.0b013e318192ccc8

Das, A., \& Fiete, I. R. (2020). Systematic errors in connectivity inferred from activity in strongly recurrent networks. In Nature Neuroscience (Vol. 23, Issue 10, pp. 1286-1296). https://doi.org/10.1038/s41593-020-0699-2

Dehaene, S., Kerszberg, M., \& Changeux, J. P. (1998). A neuronal model of a global workspace in effortful cognitive tasks. Proceedings of the National Academy of Sciences of the United States of America, 95(24), 14529-14534. https://www.ncbi.nlm.nih.gov/pubmed/9826734

Deiber, M. P., Ibañez, V., Bastuji, H., Fischer, C., \& Mauguière, F. (1989). Changes of middle latency auditory evoked potentials during natural sleep in humans. Neurology, 39(6), 806-813. https://doi.org/10.1212/wnl.39.6.806

De Salvo, S. (2015). Neurophysiological assessment for evaluating residual cognition in vegetative and minimally conscious state patients: a pilot study. Functional Neurology. https://doi.org/10.11138/FNeur/2015.30.4.237

Doerig, A., Schurger, A., \& Herzog, M. H. (2020). Hard criteria for empirical theories of consciousness. Cognitive Neuroscience, 1-22. https://doi.org/10.1080/17588928.2020.1772214

Duchateau, F.-X., Saunier, M., Larroque, B., Josseaume, J., Gauss, T., Curac, S., Wojciechowski-Bonnal, E., \& Mantz, J. (2014). Use of bispectral index to monitor the depth of sedation in mechanically ventilated patients in the prehospital setting. Emergency Medicine Journal: EMJ, 31(8), 669-672. https://doi.org/10.1136/emermed-2012-202238 
Engemann, D. A., Raimondo, F., King, J.-R., Rohaut, B., Louppe, G., Faugeras, F., Annen, J., Cassol, H., Gosseries, O., Fernandez-Slezak, D., Laureys, S., Naccache, L., Dehaene, S., \& Sitt, J. D. (2018). Robust EEG-based cross-site and cross-protocol classification of states of consciousness. Brain: A Journal of Neurology, 141(11), 3179-3192. https://doi.org/10.1093/brain/awy251

Fàbregas, N., Gambús, P. L., Valero, R., Carrero, E. J., Salvador, L., Zavala, E., \& Ferrer, E. (2004). Can bispectral index monitoring predict recovery of consciousness in patients with severe brain injury? Anesthesiology, 101(1), 43-51. https://doi.org/10.1097/00000542-200407000-00009

Fan, S. Z., Yeh, J. R., Chen, B. C., \& Shieh, J. S. (2011). Comparison of EEG approximate entropy and complexity measures of depth of anaesthesia during inhalational general anaesthesia. Journal of Medical and Biological Engineering, 31(5), 359-366. https://doi.org/10.5405/jmbe.820

Fan, S.-Z., Yeh, J.-R., Chen, B.-C., Shieh, J.-S., \& Others. (2011). Comparison of EEG approximate entropy and complexity measures of depth of anaesthesia during inhalational general anaesthesia. Journal of Medical and Biological Engineering, 31(5), 359-366. http://www.jmbe.org.tw/files/887/public/887-2681-2-PB.pdf

Farnes, N., Juel, B. E., Nilsen, A. S., Romundstad, L. G., \& Storm, J. F. (2020). Increased signal diversity/complexity of spontaneous EEG, but not evoked EEG responses, in ketamine-induced psychedelic state in humans. In bioRxiv (under revision in PLOS One) (p. 508697). https://doi.org/10.1101/508697

Fekete, T., Omer, D. B., O’Hashi, K., Grinvald, A., van Leeuwen, C., \& Shriki, O. (2018). Critical dynamics, anesthesia and information integration: Lessons from multi-scale criticality analysis of voltage imaging data. NeuroImage, 183, 919-933. https://doi.org/10.1016/j.neuroimage.2018.08.026

Ferenets, R., Lipping, T., Anier, A., Jäntti, V., Melto, S., \& Hovilehto, S. (2006). Comparison of entropy and complexity measures for the assessment of depth of sedation. IEEE Transactions on Bio-Medical Engineering, 53(6), 1067-1077. https://doi.org/10.1109/TBME.2006.873543

Finke, A., Steppacher, I., Kissler, J., \& Ritter, H. (2018). Frequency Band Variations Predict EEG Single-Trial Classification Performance in Disorder of Consciousness Patients. In 2018 40th Annual International Conference of the IEEE Engineering in Medicine and Biology Society (EMBC). https://doi.org/10.1109/embc.2018.8512719

Fuentes, R., Cortínez, L. I., Struys, M. M. R. F., Delfino, A., \& Muñoz, H. (2008). The dynamic relationship between end-tidal sevoflurane concentrations, bispectral index, and cerebral state index in children. Anesthesia and Analgesia, 107(5), 1573-1578. https://doi.org/10.1213/ane.0b013e318181ef88 
Gallup, G. G., Jr, Anderson, J. R., \& Shillito, D. J. (2002). The mirror test. The Cognitive Animal: Empirical and Theoretical Perspectives on Animal Cognition, 325-333.

http://philosophy.hku.hk/courses/cogsci/files/gallup-final.pdf

Gaskell, A. L., Hight, D. F., Winders, J., Tran, G., Defresne, A., Bonhomme, V., Raz, A., Sleigh, J. W., \& Sanders, R. D. (2017). Frontal alpha-delta EEG does not preclude volitional response during anaesthesia: prospective cohort study of the isolated forearm technique. British Journal of Anaesthesia, 119(4), 664-673. https://doi.org/10.1093/bja/aex170

Gosseries, O., Schnakers, C., Ledoux, D., Vanhaudenhuyse, A., Bruno, M.-A., Demertzi, A., Noirhomme, Q., Lehembre, R., Damas, P., Goldman, S., Peeters, E., Moonen, G., \& Laureys, S. (2011). Automated EEG entropy measurements in coma, vegetative state/unresponsive wakefulness syndrome and minimally conscious state. Functional Neurology, 26(1), 25-30. https://www.ncbi.nlm.nih.gov/pubmed/21693085

Haenggi, M., Ypparila-Wolters, H., Buerki, S., Schlauri, R., Korhonen, I., Takala, J., \& Jakob, S. M. (2009). Auditory event-related potentials, Bispectral Index, and entropy for the discrimination of different levels of sedation in intensive care unit patients. Anesthesia and Analgesia, 109(3), 807-816. https://doi.org/10.1213/ane.0b013e3181acc85d

Herrmann, C. S., Strüber, D., Helfrich, R. F., \& Engel, A. K. (2016). EEG oscillations: From correlation to causality. International Journal of Psychophysiology: Official Journal of the International Organization of Psychophysiology, 103, 12-21. https://doi.org/10.1016/j.ijpsycho.2015.02.003

Hirota, K. (2006). Special cases: ketamine, nitrous oxide and xenon. Best Practice \& Research. Clinical Anaesthesiology, 20(1), 69-79. https://doi.org/10.1016/j.bpa.2005.08.014

Höller, Y., Thomschewski, A., Bergmann, J., Kronbichler, M., Crone, J. S., Schmid, E. V., Butz, K., Höller, P., Nardone, R., \& Trinka, E. (2014). Connectivity biomarkers can differentiate patients with different levels of consciousness. Clinical Neurophysiology: Official Journal of the International Federation of Clinical Neurophysiology, 125(8), 1545-1555. https://doi.org/10.1016/j.clinph.2013.12.095

Hughes, J. R., \& John, E. R. (1999). Conventional and quantitative electroencephalography in psychiatry. The Journal of Neuropsychiatry and Clinical Neurosciences, 11(2), 190-208.

https://doi.org/10.1176/jnp.11.2.190

Jordan, D., Stockmanns, G., Kochs, E. F., \& Schneider, G. (2007). Median frequency revisited: an approach to improve a classic spectral electroencephalographic parameter for the separation of consciousness from 
unconsciousness. Anesthesiology, 107(3), 397-405. https://doi.org/10.1097/01.anes.0000278904.63884.4c

Kaskinoro, K., Maksimow, A., Långsjö, J., Aantaa, R., Jääskeläinen, S., Kaisti, K., Särkelä, M., \& Scheinin, H. (2011). Wide inter-individual variability of bispectral index and spectral entropy at loss of consciousness during increasing concentrations of dexmedetomidine, propofol, and sevoflurane. British Journal of Anaesthesia, 107(4), 573-580. https://doi.org/10.1093/bja/aer196

Kim, H. M., Shin, S. W., Yoon, J. Y., Lee, H. J., Kim, K. H., \& Baik, S. W. (2012). Effects of etomidate on bispectral index scale and spectral entropy during induction of anesthesia by means of the raw electroencephalographic and electromyographic characteristics. Korean Journal of Anesthesiology, 62(3), 230-233. https://doi.org/10.4097/kjae.2012.62.3.230

Kim, S.-P., Hwang, E., Kang, J.-H., Kim, S., \& Choi, J. H. (2012). Changes in the thalamocortical connectivity during anesthesia-induced transitions in consciousness. Neuroreport, 23(5), 294-298. https://doi.org/10.1097/WNR.0b013e3283509ba0

King, J. R., Faugeras, F., Gramfort, A., Schurger, A., El Karoui, I., Sitt, J. D., Rohaut, B., Wacongne, C., Labyt, E., Bekinschtein, T., Cohen, L., Naccache, L., \& Dehaene, S. (2013). Single-trial decoding of auditory novelty responses facilitates the detection of residual consciousness. NeuroImage, 83, 726-738. https://doi.org/10.1016/j.neuroimage.2013.07.013

Klimesch, W. (1999). EEG alpha and theta oscillations reflect cognitive and memory performance: a review and analysis. Brain Research. Brain Research Reviews, 29(2-3), 169-195. https://doi.org/10.1016/s0165-0173(98)00056-3

Koch, C., Massimini, M., Boly, M., \& Tononi, G. (2016). Neural correlates of consciousness: progress and problems. Nature Reviews. Neuroscience, 17(5), 307-321. https://doi.org/10.1038/nrn.2016.22

Lavazza, A., \& Massimini, M. (2018). Cerebral organoids: ethical issues and consciousness assessment. Journal of Medical Ethics, 44(9), 606-610. https://doi.org/10.1136/medethics-2017-104555

Lee, M., Baird, B., Gosseries, O., Nieminen, J. O., Boly, M., Postle, B. R., Tononi, G., \& Lee, S. W. (2019). Connectivity differences between consciousness and unconsciousness in non-rapid eye movement sleep: a TMS-EEG study. Scientific Reports, 9(1). https://doi.org/10.1038/s41598-019-41274-2

Lee, U., Kim, S., Noh, G.-J., Choi, B.-M., Hwang, E., \& Mashour, G. A. (2009). The directionality and functional organization of frontoparietal connectivity during consciousness and anesthesia in humans. Consciousness and Cognition, 18(4), 1069-1078. https://doi.org/10.1016/j.concog.2009.04.004 
Leslie, K. (2017). Awareness and Dreaming During TIVA. In A. R. Absalom \& K. P. Mason (Eds.), Total Intravenous Anesthesia and Target Controlled Infusions: A Comprehensive Global Anthology (pp. 783-796). Springer International Publishing. https://doi.org/10.1007/978-3-319-47609-4_44

Leslie, K., Skrzypek, H., Paech, M. J., Kurowski, I., \& Whybrow, T. (2007). Dreaming during Anesthesia and Anesthetic Depth in Elective Surgery PatientsA Prospective Cohort Study. Anesthesiology, 106(1), 33-42. http://anesthesiology.pubs.asahq.org/article.aspx?articleid=1922745

Leslie, K., Sleigh, J., Paech, M. J., Voss, L., Lim, C. W., \& Sleigh, C. (2009). Dreaming and electroencephalographic changes during anesthesia maintained with propofol or desflurane. Anesthesiology, 111(3), 547-555. https://doi.org/10.1097/ALN.0b013e3181adf768

Liang, Z., Liang, S., Wang, Y., Ouyang, G., \& Li, X. (2015). Tracking the coupling of two electroencephalogram series in the isoflurane and remifentanil anesthesia. Clinical Neurophysiology: Official Journal of the International Federation of Clinical Neurophysiology, 126(2), 412-422.

https://doi.org/10.1016/j.clinph.2014.05.012

Loomis, A. L., Harvey, E. N., \& Hobart, G. A. (1937). Cerebral states during sleep, as studied by human brain potentials. In Journal of Experimental Psychology (Vol. 21, Issue 2, pp. 127-144).

https://doi.org/10.1037/h0057431

Luauté, J., Maucort-Boulch, D., Tell, L., Quelard, F., Sarraf, T., Iwaz, J., Boisson, D., \& Fischer, C. (2010). Long-term outcomes of chronic minimally conscious and vegetative states. Neurology, 75(3), 246-252. https://doi.org/10.1212/WNL.0b013e3181e8e8df

Lu, C. H., Chen, J. L., Wu, C. T., Liaw, W. J., Yeh, C. C., Cherng, C. H., \& Wong, C. S. (2010). Effect of Epidural Neuraxial Blockade-dependent Sedation on the Ramsay Sedation Scale and the Composite Auditory Evoked Potentials Index in Surgical Intensive Care Patients. Journal of the Formosan Medical Association = Taiwan Yi Zhi, 109(8), 589-595. https://doi.org/10.1016/S0929-6646(10)60096-4

Lu, C. H., Man, K. M., Ou-Yang, H. Y., Chan, S. M., Ho, S. T., Wong, C. S., \& Liaw, W. J. (2008). Composite auditory evoked potential index versus bispectral index to estimate the level of sedation in paralyzed critically ill patients: A prospective observational study. Anesthesia and Analgesia, 107(4), 1290-1294. https://doi.org/10.1213/ane.0b013e31818061ae

Lysakowski, C., Elia, N., Czarnetzki, C., Dumont, L., Haller, G., Combescure, C., \& Tramèr, M. R. (2009). Bispectral and spectral entropy indices at propofol-induced loss of consciousness in young and elderly 
patients. British Journal of Anaesthesia, 103(3), 387-393. https://doi.org/10.1093/bja/aep162

Maksimow, A., Silfverhuth, M., Långsjö, J., Kaskinoro, K., Georgiadis, S., Jääskeläinen, S., \& Scheinin, H. (2014). Directional connectivity between frontal and posterior brain regions is altered with increasing concentrations of propofol. PloS One, 9(11), e113616. https://doi.org/10.1371/journal.pone.0113616

Mashour, G. A., \& Hudetz, A. G. (2018). Neural Correlates of Unconsciousness in Large-Scale Brain Networks. Trends in Neurosciences, 41(3), 150-160. https://doi.org/10.1016/j.tins.2018.01.003

Mashour, G. A., Roelfsema, P., Changeux, J.-P., \& Dehaene, S. (2020). Conscious Processing and the Global Neuronal Workspace Hypothesis. Neuron, 105(5), 776-798. https://doi.org/10.1016/j.neuron.2020.01.026

Massimini, M., Boly, M., Casali, A., Rosanova, M., \& Tononi, G. (2009). A perturbational approach for evaluating the brain's capacity for consciousness. Progress in Brain Research, 177, 201-214. https://doi.org/10.1016/S0079-6123(09)17714-2

Mediano, P. A. M., Seth, A. K., \& Barrett, A. B. (2018). Measuring Integrated Information: Comparison of Candidate Measures in Theory and Simulation. Entropy, 21(1), 17. https://doi.org/10.3390/e21010017

Merker, B. (2007). Consciousness without a cerebral cortex: a challenge for neuroscience and medicine. The Behavioral and Brain Sciences, 30(1), 63-81; discussion 81-134.

https://doi.org/10.1017/S0140525X07000891

Michel, M., Beck, D., Block, N., Blumenfeld, H., Brown, R., Carmel, D., Carrasco, M., Chirimuuta, M., Chun, M., Cleeremans, A., Dehaene, S., Fleming, S. M., Frith, C., Haggard, P., He, B. J., Heyes, C., Goodale, M. A., Irvine, L., Kawato, M., .. Yoshida, M. (2019). Opportunities and challenges for a maturing science of consciousness. Nature Human Behaviour, 3(2), 104-107. https://doi.org/10.1038/s41562-019-0531-8

Nagel, T. (1974). What is it like to be a bat? The Philosophical Review, 83(4), 435-450. https://www.jstor.org/stable/2183914

Nicolaou, N., Georgiou, J., \& Absalom, A. R. (2014). Global field synchrony during general anaesthesia. British Journal of Anaesthesia, 112(3), 529-539. https://doi.org/10.1093/bja/aet350

Nielsen, T. A. (2000). A review of mentation in REM and NREM sleep: "Covert" REM sleep as a possible reconciliation of two opposing models. The Behavioral and Brain Sciences, 23(6), 851-866. https://doi.org/10.1017/S0140525X0000399X

Nieuwenhuijs, D., Coleman, E. L., Douglas, N. J., Drummond, G. B., \& Dahan, A. (2002). Bispectral Index Values and Spectral Edge Frequency at Different Stages of Physiologic Sleep. Anesthesia \& Analgesia, 
94(1), 125-129. https://doi.org/10.1097/00000539-200201000-00024

Nilsen, A. S., Juel, B. E., \& Storm, J. F. (2019). Measures of states of consciousness during attentional and cognitive load. In bioRxiv (p. 586149). https://doi.org/10.1101/586149

Ni Mhuircheartaigh, R., Warnaby, C., Rogers, R., Jbabdi, S., \& Tracey, I. (2013). Slow-Wave Activity Saturation and Thalamocortical Isolation During Propofol Anesthesia in Humans. Science Translational Medicine, 5(208), 208ra148-ra208ra148. https://doi.org/10.1126/scitranslmed.3006007

Noreika, V., Jylhänkangas, L., Móró, L., Valli, K., Kaskinoro, K., Aantaa, R., Scheinin, H., \& Revonsuo, A. (2011a). Consciousness lost and found: subjective experiences in an unresponsive state. Brain and Cognition, 77(3), 327-334. https://doi.org/10.1016/j.bandc.2011.09.002

Noreika, V., Jylhänkangas, L., Móró, L., Valli, K., Kaskinoro, K., Aantaa, R., Scheinin, H., \& Revonsuo, A. (2011b). Consciousness lost and found: subjective experiences in an unresponsive state. Brain and Cognition, 77(3), 327-334. https://doi.org/10.1016/j.bandc.2011.09.002

Northoff, G. (2017). "Paradox of slow frequencies" - Are slow frequencies in upper cortical layers a neural predisposition of the level/state of consciousness (NPC)? Consciousness and Cognition, 54, 20-35. https://doi.org/10.1016/j.concog.2017.03.006

Odegaard, B., Knight, R. T., \& Lau, H. (2017). Should a Few Null Findings Falsify Prefrontal Theories of Conscious Perception? The Journal of Neuroscience: The Official Journal of the Society for Neuroscience, 37(40), 9593-9602. https://doi.org/10.1523/JNEUROSCI.3217-16.2017

Owen, A. M., Coleman, M. R., Boly, M., Davis, M. H., Laureys, S., \& Pickard, J. D. (2006). Detecting awareness in the vegetative state. Science, 313(5792), 1402. https://doi.org/10.1126/science.1130197

Pagel, J. F. (2012). The synchronous electrophysiology of conscious states. Dreaming: Journal of the Association for the Study of Dreams, 22(3), 173-191. https://doi.org/10.1037/a0029659

Pilge, S., Blum, J., Kochs, E. F., Schöniger, S. A., Kreuzer, M., \& Schneider, G. (2011). Does the cerebral state index separate consciousness from unconsciousness? Anesthesia and Analgesia, 113(6), 1403-1410. https://doi.org/10.1213/ANE.0b013e31823007cd

Pincus, S. M. (1991). Approximate entropy as a measure of system complexity. Proceedings of the National Academy of Sciences of the United States of America, 88(6), 2297-2301. https://doi.org/10.1073/pnas.88.6.2297

Railo, H., Koivisto, M., \& Revonsuo, A. (2011). Tracking the processes behind conscious perception: a review 
of event-related potential correlates of visual consciousness. Consciousness and Cognition, 20(3), 972-983. https://doi.org/10.1016/j.concog.2011.03.019

Rees, G., Kreiman, G., \& Koch, C. (2002). Neural correlates of consciousness in humans. Nature Reviews. Neuroscience, 3(4), 261-270. https://doi.org/10.1038/nrn783

Rizkallah, J., Annen, J., Modolo, J., Gosseries, O., Benquet, P., Mortaheb, S., Amoud, H., Cassol, H., Mheich, A., Thibaut, A., Chatelle, C., Hassan, M., Panda, R., Wendling, F., \& Laureys, S. (2019). Decreased integration of EEG source-space networks in disorders of consciousness. NeuroImage. Clinical, 23, 101841. https://doi.org/10.1016/j.nicl.2019.101841

Sanchez-Vives, M. V., Massimini, M., \& Mattia, M. (2017). Shaping the Default Activity Pattern of the Cortical Network. Neuron, 94(5), 993-1001. https://doi.org/10.1016/j.neuron.2017.05.015

Sanders, R. D., Banks, M. I., Darracq, M., Moran, R., Sleigh, J., Gosseries, O., Bonhomme, V., Brichant, J. F., Rosanova, M., Raz, A., Tononi, G., Massimini, M., Laureys, S., \& Boly, M. (2018). Propofol-induced unresponsiveness is associated with impaired feedforward connectivity in cortical hierarchy. British Journal of Anaesthesia, 121(5), 1084-1096. https://doi.org/10.1016/j.bja.2018.07.006

Sanders, R. D., Tononi, G., Laureys, S., \& Sleigh, J. W. (2012). Unresponsiveness $\neq$ unconsciousness. Anesthesiology, 116(4), 946-959. https://doi.org/10.1097/ALN.0b013e318249d0a7

Sarasso, S., Boly, M., Napolitani, M., Gosseries, O., Charland-Verville, V., Casarotto, S., Rosanova, M., Casali, A. G., Brichant, J.-F., Boveroux, P., Rex, S., Tononi, G., Laureys, S., \& Massimini, M. (2015).

Consciousness and Complexity during Unresponsiveness Induced by Propofol, Xenon, and Ketamine. Current Biology: CB, 25(23), 3099-3105. https://doi.org/10.1016/j.cub.2015.10.014

Schartner, M. M., Carhart-Harris, R. L., Barrett, A. B., Seth, A. K., \& Muthukumaraswamy, S. D. (2017). Increased spontaneous MEG signal diversity for psychoactive doses of ketamine, LSD and psilocybin. Scientific Reports, 7, 46421. https://doi.org/10.1038/srep46421

Schartner, M. M., Pigorini, A., Gibbs, S. A., Arnulfo, G., Sarasso, S., Barnett, L., Nobili, L., Massimini, M., Seth, A. K., \& Barrett, A. B. (2017). Global and local complexity of intracranial EEG decreases during NREM sleep. Neuroscience of Consciousness, 2017(1), niw022. https://doi.org/10.1093/nc/niw022

Schartner, M., Seth, A., Noirhomme, Q., Boly, M., Bruno, M. A., Laureys, S., \& Barrett, A. (2015). Complexity of multi-dimensional spontaneous EEG decreases during propofol induced general anaesthesia. PloS One, 10(8). https://doi.org/10.1371/journal.pone.0133532 
Scheel, A. M., Schijen, M., \& Lakens, D. (2020). An excess of positive results: Comparing the standard Psychology literature with Registered Reports. https://doi.org/10.31234/osf.io/p6e9c

Schnakers, C., Ledoux, D., Majerus, S., Damas, P., Damas, F., Lambermont, B., Lamy, M., Boly, M., Vanhaudenhuyse, A., Moonen, G., \& Laureys, S. (2008). Diagnostic and prognostic use of bispectral index in coma, vegetative state and related disorders. Brain Injury: [BI], 22(12), 926-931. https://doi.org/10.1080/02699050802530565

Schnakers, C., Vanhaudenhuyse, A., Giacino, J., Ventura, M., Boly, M., Majerus, S., Moonen, G., \& Laureys, S. (2009). Diagnostic accuracy of the vegetative and minimally conscious state: clinical consensus versus standardized neurobehavioral assessment. BMC Neurology, 9, 35. https://doi.org/10.1186/1471-2377-9-35

Schuller, P. J., Newell, S., Strickland, P. A., \& Barry, J. J. (2015). Response of bispectral index to neuromuscular block in awake volunteers. British Journal of Anaesthesia, 115 Suppl 1(suppl 1), i95-i103. https://doi.org/10.1093/bja/aev072

Schwartze, M., Farrugia, N., \& Kotz, S. A. (2013). Dissociation of formal and temporal predictability in early auditory evoked potentials. Neuropsychologia, 51(2), 320-325. https://doi.org/10.1016/j.neuropsychologia.2012.09.037

Schwitzgebel, E. (2008). The unreliability of naive introspection. The Philosophical Review. https://read.dukeupress.edu/the-philosophical-review/article-abstract/117/2/245/2787?casa_token=7NO4joh9bIAAAAA:K98tx2WYFeOqwImgbV01CZGXTt5cfWNjn47IhEp-v-7O1nimUpFwWSIXuNuRyyAKs-z DwQ2tBg

Sergent, C., \& Dehaene, S. (2004). Neural processes underlying conscious perception: Experimental findings and a global neuronal workspace framework. Journal of Physiology, Paris, 98(4-6 SPEC. ISS.), 374-384. https://doi.org/10.1016/j.jphysparis.2005.09.006

Seth, A. K. (2010). The grand challenge of consciousness. Frontiers in Psychology, 1, 5. https://doi.org/10.3389/fpsyg.2010.00005

Seth, A. K., Dienes, Z., Cleeremans, A., Overgaard, M., \& Pessoa, L. (2008). Measuring consciousness: relating behavioural and neurophysiological approaches. Trends in Cognitive Sciences, 12(8), 314-321. https://doi.org/10.1016/j.tics.2008.04.008

Shalbaf, R., Behnam, H., Sleigh, J. W., Steyn-Ross, A., \& Voss, L. J. (2013). Monitoring the depth of anesthesia using entropy features and an artificial neural network. Journal of Neuroscience Methods, 218(1), 17-24. 
https://doi.org/10.1016/j.jneumeth.2013.03.008

Shepherd, J., Jones, J., Frampton, G. K., Bryant, J., Baxter, L., \& Cooper, K. (2013). Clinical effectiveness and cost-effectiveness of depth of anaesthesia monitoring (E-Entropy, Bispectral Index and Narcotrend): A systematic review and economic evaluation. Health Technology Assessment, 17(34), 1-264. https://doi.org/10.3310/hta17340

Shibata, S., Imota, T., Shigeomi, S., Sato, W., \& Enzan, K. (2005). Use of the bispectral index during the early postresuscitative phase after out-of-hospital cardiac arrest. Journal of Anesthesia, 19(3), 243-246. https://doi.org/10.1007/s00540-005-0317-1

Shin, T. J., Cho, D., Ham, J., Choi, D. H., Kim, S., Jeong, S., Kim, H. I., Kim, J. G., \& Lee, B. (2016). Changes in thalamo-frontal interaction under different levels of anesthesia in rats. Neuroscience Letters, 627, 18-23. https://doi.org/10.1016/j.neulet.2016.05.045

Siclari, F., Bernardi, G., Cataldi, J., \& Tononi, G. (2018). Dreaming in NREM Sleep: A High-Density EEG Study of Slow Waves and Spindles. The Journal of Neuroscience: The Official Journal of the Society for Neuroscience, 38(43), 9175-9185. https://doi.org/10.1523/JNEUROSCI.0855-18.2018

Sitt, J. D., King, J. R., El Karoui, I., Rohaut, B., Faugeras, F., Gramfort, A., Cohen, L., Sigman, M., Dehaene, S., \& Naccache, L. (2014). Large scale screening of neural signatures of consciousness in patients in a vegetative or minimally conscious state. Brain: A Journal of Neurology, 137(8), 2258-2270. https://doi.org/10.1093/brain/awu141

Stefan, S., Schorr, B., Lopez-Rolon, A., Kolassa, I.-T., Shock, J. P., Rosenfelder, M., Heck, S., \& Bender, A. (2018). Consciousness Indexing and Outcome Prediction with Resting-State EEG in Severe Disorders of Consciousness. Brain Topography, 31(5), 848-862. https://doi.org/10.1007/s10548-018-0643-x

Steriade, M., Gloor, P., Llinás, R. R., Lopes da Silva, F. H., \& Mesulam, M.-M. (1990). Basic mechanisms of cerebral rhythmic activities. Electroencephalography and Clinical Neurophysiology, 76(6), 481-508. https://doi.org/10.1016/0013-4694(90)90001-Z

Sternbach, G. L. (2000). The Glasgow coma scale. The Journal of Emergency Medicine, 19(1), 67-71. https://doi.org/10.1016/s0736-4679(00)00182-7

Supp, G. G., Higgen, F. L., Hipp, J. F., Engel, A. K., \& Siegel, M. (2018). Mid-Latency Auditory Evoked Potentials Differentially Predict Sedation and Drug Level Under Opioid and Hypnotic Agents. Frontiers in Pharmacology, 9, 1427. https://doi.org/10.3389/fphar.2018.01427 
Tavakoli, P., Varma, S., \& Campbell, K. (2018). Highly relevant stimuli may passively elicit processes associated with consciousness during the sleep onset period. Consciousness and Cognition, 58, 60-74. https://doi.org/10.1016/j.concog.2017.10.012

Thakor, N. V., \& Tong, S. (2004). Advances in quantitative electroencephalogram analysis methods. Annual Review of Biomedical Engineering, 6, 453-495. https://doi.org/10.1146/annurev.bioeng.5.040202.121601

Tononi, G. (2004). An information integration theory of consciousness. BMC Neuroscience, 5(1), 42. https://doi.org/10.1186/1471-2202-5-42

Tsurukiri, J., Nagata, K., Okita, T., \& Oomura, T. (2013). Middle latency auditory-evoked potential index for predicting the degree of consciousness of comatose patients in EDs. The American Journal of Emergency Medicine, 31(11), 1556-1559. https://doi.org/10.1016/j.ajem.2013.06.012

Valkenburg, A. J., De Leeuw, T. G., Tibboel, D., \& Weber, F. (2009). Lower bispectral index values in children who are intellectually disabled. Anesthesia and Analgesia, 109(5), 1428-1433.

https://doi.org/10.1213/01ANE.0b013e3181ba43b2

Vanhaudenhuyse, A., Charland-Verville, V., Thibaut, A., Chatelle, C., Tshibanda, J.-F. L., Maudoux, A., Faymonville, M.-E., Laureys, S., \& Gosseries, O. (2018). Conscious While Being Considered in an Unresponsive Wakefulness Syndrome for 20 Years. Frontiers in Neurology, 9, 671. https://doi.org/10.3389/fneur.2018.00671

Viertio-Oja, H., Maja, V., Sarkela, M., Talja, P., Tenkanen, N., Tolvanen-Laakso, H., Paloheimo, M., Vakkuri, A., Yli-Hankala, A., \& Merilainen, P. (2004). Description of the Entropytm algorithm as applied in the Datex-Ohmeda S/5tm Entropy Module. Acta Anaesthesiologica Scandinavica, 48(2), 154-161. https://doi.org/10.1111/j.0001-5172.2004.00322.x

Warnaby, C. E., Sleigh, J. W., Hight, D., Jbabdi, S., \& Tracey, I. (2017). Investigation of Slow-wave Activity Saturation during Surgical Anesthesia Reveals a Signature of Neural Inertia in Humans. Anesthesiology, 127(4), 645-657. https://doi.org/10.1097/ALN.0000000000001759

Wenzel, M., Han, S., Smith, E. H., Hoel, E., Greger, B., House, P. A., \& Yuste, R. (2019). Reduced Repertoire of Cortical Microstates and Neuronal Ensembles in Medically Induced Loss of Consciousness. Cell Systems. https://doi.org/10.1016/j.cels.2019.03.007

Yppärilä, H., Korhonen, I., Tarvainen, M., Musialowicz, T., Jakob, S. M., \& Partanen, J. (2004). N100 auditory potential and electroencephalogram discriminate propofol-induced sedation levels. Journal of Clinical 
Monitoring and Computing, 18(3), 163-170. https://doi.org/10.1023/B:JOCM.0000042921.47988.bf

Yurgil, K. A., \& Golob, E. J. (2010). Neural activity before and after conscious perception in dichotic listening. Neuropsychologia, 48(10), 2952-2958. https://doi.org/10.1016/j.neuropsychologia.2010.06.004

Owen, A. M., Schiff, N. D., \& Laureys, S. (2009). A new era of coma and consciousness science. Progress in brain research, 177, 399-411.

Schwitzgebel, E. (2008). The unreliability of naive introspection. Philosophical Review, 117(2), 245-273. https://doi.org/10.1215/00318108-2007-037 


\section{FIGURES}

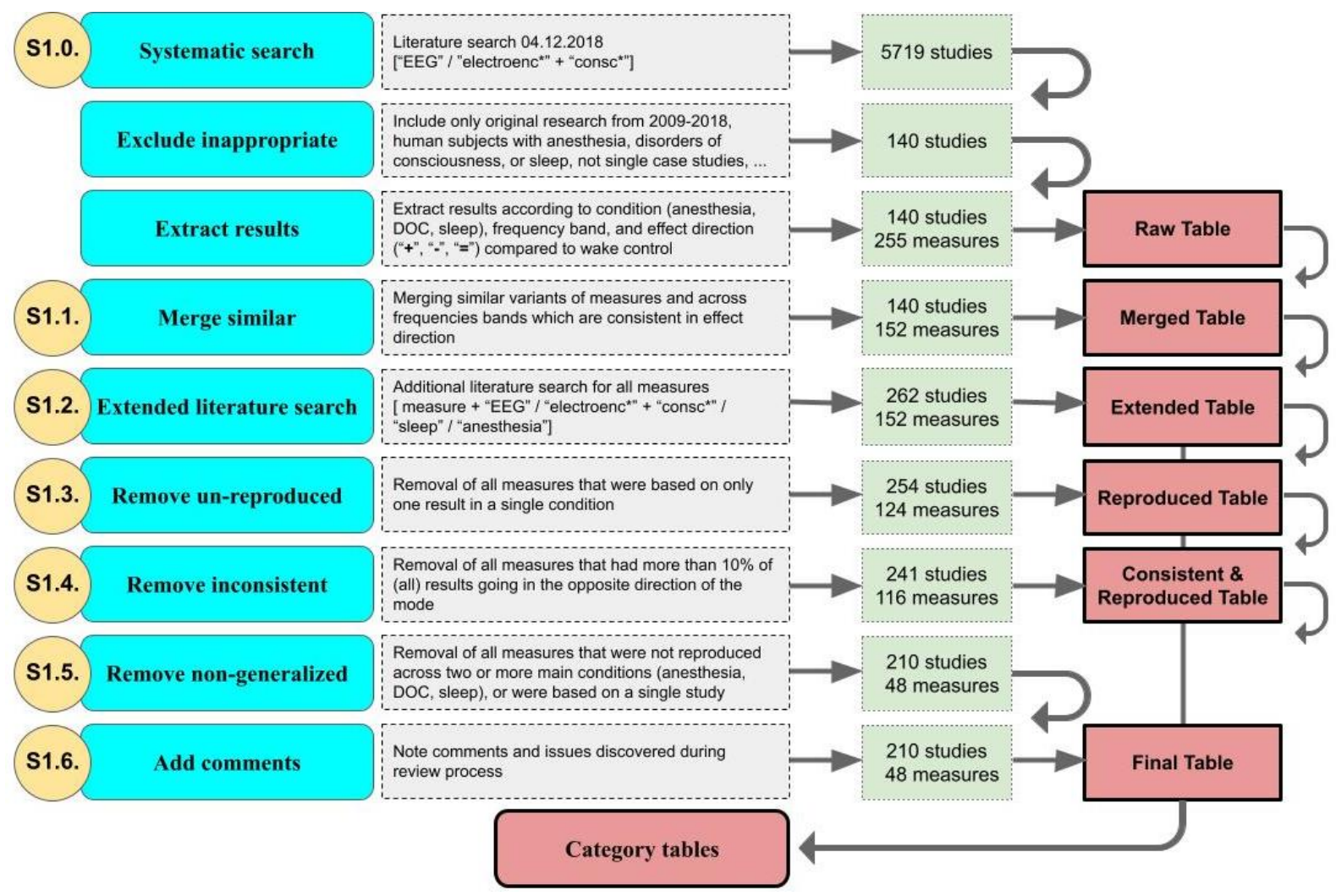

Figure 1: 6-step literature search procedure. The whole procedure that was performed for the systematic literature search and for filtering out measures included six key steps resulting in six tables. The full tables can be seen in the supplementary data (SDAT: Supplementary_Measures and Supplementary_Categories), and the details about each step can be seen in the supplementary material (Suppl. 1). The remaining measures after the filtering steps "S1.2", "S1.3", "S1.4", and "S1.6", were used to produce the category table (Suppl. 2). 


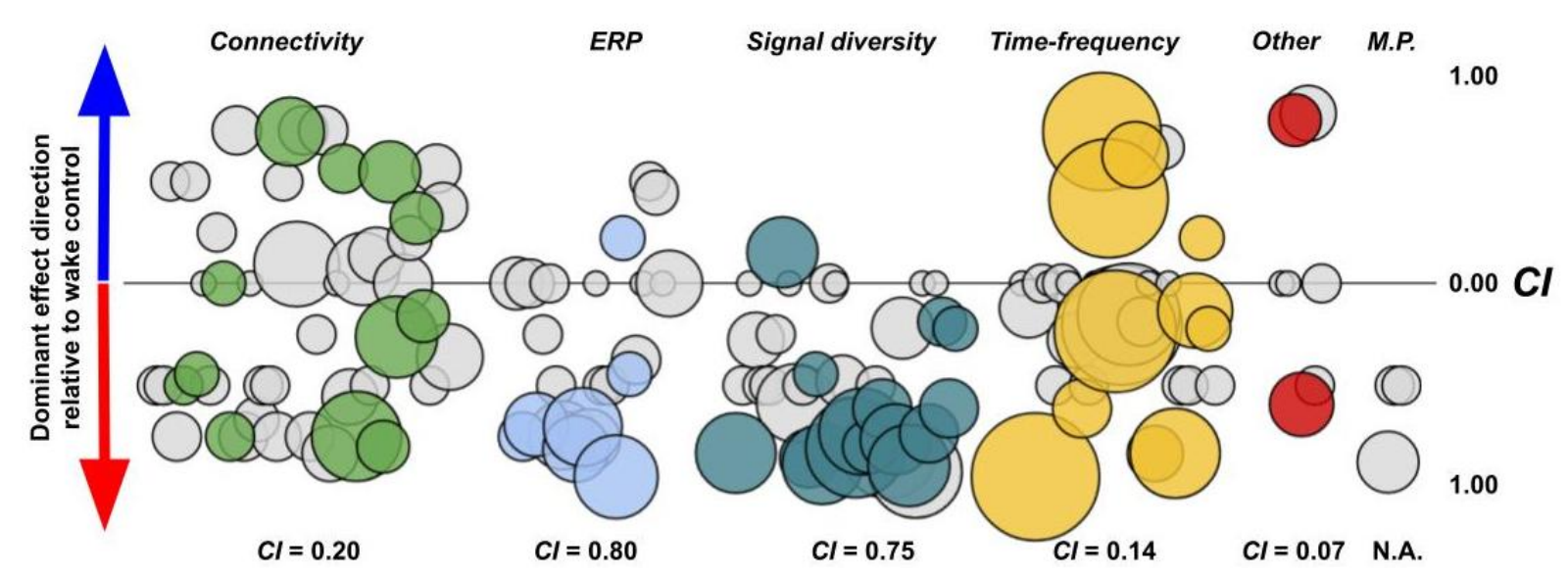

Figure 2. Overview of effect directionality of measures. Each bubble represents a single measure with size determined by the number of results registered and vertical position determined by the overall consistency of effect directionality (consistency index; $C I$ ) with below the $\mathrm{x}$-axis being a decrease relative to wake controls, and above the $\mathrm{x}$-axis being an increase relative to wake control.. Light grey bubbles represent measures that were removed in the filtering process (see Suppl. 1) with $C I$ determined at filtering step S1.2. Colored bubbles are measures that remained after the filtering process, with $C I$ determined at filtering step S1.6. $C I$ for categories, presented as numbers along the bottom of the figure, was determined at S1.6. The category multi-parameter (M.P.) had no measures surviving the filtering process and therefore has no estimate of consistency available. 


\section{TABLES}

Table 1. Overview of results for categories of measures

\begin{tabular}{|c|c|c|c|c|c|c|c|c|c|c|c|c|c|}
\hline \multirow[b]{2}{*}{ Category } & \multicolumn{3}{|c|}{$\begin{array}{c}\text { Results } \\
\text { Anesthesia }\end{array}$} & \multicolumn{3}{|c|}{$\begin{array}{l}\text { Results } \\
\text { DOC }\end{array}$} & \multicolumn{3}{|c|}{$\begin{array}{l}\text { Results } \\
\text { Sleep }\end{array}$} & \multirow[t]{2}{*}{$\begin{array}{c}\text { Results } \\
\text { Total }\end{array}$} & \multirow[t]{2}{*}{$\mathrm{Cl}$} & \multirow[t]{2}{*}{$N_{M}$} & \multirow[t]{2}{*}{$\mathbf{N}_{s}$} \\
\hline & + & - & $=$ & + & - & $=$ & + & - & $=$ & & & & \\
\hline \multicolumn{14}{|c|}{ Extended Table, S1.2 } \\
\hline Connectivity & 25 & 42 & 28 & 22 & 55 & 24 & 19 & 7 & 6 & 228 & 0.17 & 36 & 61 \\
\hline$E R P$ & 1 & 34 & 1 & 4 & 46 & 24 & 6 & 8 & 4 & 128 & 0.6 & 26 & 67 \\
\hline Multi-param. & & 12 & & & & & & & & 12 & 0.92 & 4 & 10 \\
\hline Other & 7 & 4 & & 4 & 7 & & 1 & & 3 & 26 & 0.04 & 4 & 11 \\
\hline Signal div. & 4 & 148 & 21 & & 42 & 29 & 3 & 27 & 5 & 279 & 0.75 & 33 & 83 \\
\hline Time-freq. & 80 & 122 & 58 & 19 & 32 & 43 & 9 & 19 & 3 & 385 & 0.17 & 28 & 129 \\
\hline \multicolumn{14}{|c|}{ Final Table, S1.6 } \\
\hline Connectivity & 2 & 15 & 14 & 6 & 19 & 9 & 15 & 7 & 4 & 91 & 0.2 & 9 & 29 \\
\hline$E R P$ & 1 & 32 & & 2 & 25 & 3 & 1 & 8 & 3 & 75 & 0.8 & 8 & 54 \\
\hline \multicolumn{14}{|l|}{ Multi-param. } \\
\hline Other & & & & 4 & 6 & & 1 & & 3 & 14 & 0.07 & 1 & 8 \\
\hline Signal div. & 1 & 82 & 10 & & 25 & 18 & 3 & 26 & 5 & 170 & 0.75 & 15 & 60 \\
\hline Time-freq. & 57 & 87 & 34 & 16 & 22 & 27 & 9 & 10 & 3 & 265 & 0.14 & 8 & 111 \\
\hline
\end{tabular}

Summary overview of the six categories of measures, at each step of the filtering process. For the full version of the table, see supplementary data (SDAT: Supplementary_Categories), for more details on how it was constructed, see Suppl. 2, and for how to read the supplementary datasheet table, see Suppl. 5. Abbreviations:,,$+-=$ : number of significant increases, decreases, and non-significant changes, compared to wake control; DOC: disorders of consciousness; Total: total number of results/findings; $\boldsymbol{C I}$ : consistency index (overall consistency of effect directionality compared to wakefulness, see section 2.2 and Suppl. 3); $N_{M}$ : number of unique measures (frequency band variants not taken into account); $\boldsymbol{N}_{S}$ : number of unique studies; S1.2: extended table including all measures and studies (see Suppl. S1.2); S1.6: final table after all filtering steps (see Suppl. S1.6); Connectivity: connectivity-based measures; ERP: event-related potential and perturbation-based measures; Multi-param.: measures based on multiple parameters; Other: measures not fitting the other categories; Signal div.: signal diversity-based measures; Time-freq.: time-frequency-based measures. 
Table 2. Category consistency over frequency bands

\begin{tabular}{llcccccc}
\hline Step & Category & delta & theta & alpha & beta & gamma & broadband \\
\hline $\mathbf{S 1 . 2}$ & Overall & $\mathbf{0 . 4 1}$ & $\mathbf{0 . 2 8}$ & $\mathbf{0 . 4 3}$ & $\mathbf{0 . 4 2}$ & $\mathbf{0 . 4 7}$ & $\mathbf{0 . 6 2}$ \\
& Connectivity & 0.01 & 0.05 & 0.33 & 0.26 & 0.39 & 0.37 \\
& Time-frequency & 0.44 & 0.23 & 0.22 & 0.27 & 0.25 & 0.68 \\
& Signal diversity & 0.71 & 0.71 & 0.68 & 0.72 & 0.72 & 0.75 \\
& ERP & 0.89 & 0.65 & 0.73 & 0.82 & 0.72 & 0.56 \\
& Multi-parameter & 0.50 & 0.50 & 0.75 & 0.75 & & 0.88 \\
& Other & 0.91 & 0.91 & 0.66 & 0.50 & 0.83 & 0.37 \\
$\mathbf{S 1 . 6}$ & & & & & & \\
& Overall & $\mathbf{0 . 5 8}$ & $\mathbf{0 . 3 7}$ & $\mathbf{0 . 4 0}$ & $\mathbf{0 . 5 1}$ & $\mathbf{0 . 4 9}$ & $\mathbf{0 . 7 1}$ \\
& Connectivity & 0.30 & 0.04 & 0.22 & 0.28 & 0.26 & 0.52 \\
& Time-frequency & 0.54 & 0.29 & 0.22 & 0.38 & 0.38 & 0.70 \\
& Signal diversity & 0.79 & 0.79 & 0.74 & 0.81 & 0.81 & 0.75 \\
& ERP & 0.86 & 0.81 & 0.78 & 0.78 & 0.76 & 0.77 \\
& Multi-parameter & & & & & & \\
Other & 0.80 & 0.80 & 0.59 & 0.59 & & 0.80 \\
\hline
\end{tabular}

All values represent overall consistency of effect directionality compared to wakefulness (consistency index; CI; see section 2.2. and Suppl. 3), for each category at each filtering step and each frequency band. For the full version of the table, see supplementary data (SDAT: Supplementary_Categories), for more details on how it was constructed, see Suppl. 2, and for how to read the supplementary datasheet table, see Suppl. 5. Abbreviations: S1.2: extended table including all measures and studies (see Suppl. S1.2); S1.6: final table after all filtering steps (see Suppl. S1.6); Connectivity: connectivity-based measures; ERP: event-related potential and perturbational-based measures; Multi-parameter: measures based on multiple parameters; Other: measures not fitting in the other categories; Signal diversity: signal diversity based measures; Time-frequency: measures based on the time-frequency domain. 
Table 3. Overview of individual EEG based measures of consciousness

\begin{tabular}{|c|c|c|c|c|c|c|c|c|c|c|c|c|c|c|}
\hline \multirow[b]{2}{*}{ Measure } & \multirow[b]{2}{*}{ Band } & \multirow[b]{2}{*}{ Cat. } & \multicolumn{3}{|c|}{$\begin{array}{c}\text { Results } \\
\text { Anesthesia }\end{array}$} & \multicolumn{3}{|c|}{$\begin{array}{l}\text { Results } \\
\text { DOC }\end{array}$} & \multicolumn{3}{|c|}{$\begin{array}{l}\text { Results } \\
\text { Sleep }\end{array}$} & \multirow[t]{2}{*}{$\begin{array}{c}\text { Results } \\
\text { Total }\end{array}$} & \multirow[t]{2}{*}{$\mathrm{Cl}$} & \multirow[t]{2}{*}{ Comment } \\
\hline & & & + & - & $=$ & + & - & $=$ & + & & $=$ & & & \\
\hline MLAEPi\&AAI & bb & ERP & & 18 & & & 1 & & & & & 19 & 0.95 & HV, EMG, O \\
\hline BIS & bb & TF & & 52 & 2 & & & & & 2 & & 56 & 0.95 & IFT, HV, EMG, PG \\
\hline SpEn & bb & SD & & 14 & & & 2 & & & 1 & 1 & 18 & 0.89 & EMG \\
\hline LZc & bb & SD & & 10 & 1 & & 2 & & & 4 & & 17 & 0.89 & \\
\hline SEF95 & bb & TF & & 13 & 2 & & 5 & & & 2 & 1 & 23 & 0.83 & \\
\hline ApEn & bb & SD & & 11 & & & 2 & 2 & & 2 & & 17 & 0.83 & \\
\hline AEP N100 & $\delta-\beta$ & ERP & & 3 & & & 4 & & & 2 & 1 & 10 & 0.81 & \\
\hline PeEn & $b b, a b$ & SD & & 13 & 1 & & 8 & 4 & & 4 & & 30 & 0.81 & IFT \\
\hline SaEn & bb & SD & & 6 & & & & & & 4 & 2 & 12 & 0.76 & \\
\hline MI & $b b, a b$ & $\mathrm{C}$ & & 8 & 3 & & 10 & 2 & & & & 23 & 0.75 & ROC \\
\hline ס-Power & $\delta$ & TF & 20 & & 6 & 11 & & 5 & 4 & & & 46 & 0.74 & IFT, PG \\
\hline AEP P3a & bb & ERP & & & & & 8 & 2 & & 1 & & 11 & 0.74 & \\
\hline GFS & $\boldsymbol{\theta}, \boldsymbol{\alpha}, \boldsymbol{\beta}, \boldsymbol{\gamma}$ & C & & 1 & & & & & 10 & & & 11 & 0.74 & \\
\hline $\mathrm{PCl}$ & bb & SD & & 7 & 2 & & 7 & 2 & & 2 & 1 & 21 & 0.73 & \\
\hline ASSR & bb, $\alpha-\gamma$ & ERP & 1 & 7 & & & 4 & & 1 & 3 & & 16 & 0.7 & HV \\
\hline bb-Power & bb & TF & 7 & & 1 & & & 2 & & & & 10 & 0.63 & ROC \\
\hline $\boldsymbol{\theta}$-Power & $\boldsymbol{\theta}$ & TF & 16 & 1 & 9 & 5 & 2 & 12 & 2 & & & 47 & 0.42 & IFT, PG \\
\hline PLI & $\mathbf{b b}, \boldsymbol{\alpha}, \boldsymbol{\beta}, \gamma$ & $\mathrm{C}$ & & 2 & 6 & & 3 & 5 & 1 & 1 & & 18 & 0.26 & o \\
\hline$\alpha$-Power & $\alpha$ & TF & & 11 & 10 & & 11 & & & 4 & & 50 & 0.24 & IFT, ROC, PG \\
\hline DFA & bb & SD & 1 & 2 & 5 & & & & 3 & & 1 & 12 & 0.15 & \\
\hline TEP Power & $a b$ & TF & & 2 & 2 & & 2 & 4 & 2 & & 2 & 14 & 0.13 & DR \\
\hline
\end{tabular}

Summary overview of the 21 EEG measures that were not excluded by the filtering process, and which had at least 10 total results in the table. The overview shows the number of results and which effect direction (compared to the wake condition) for each measure and each major condition. In addition, comments that might impact the validity of a measure are noted. For a full version of the table, see supplementary datasheet: SDAT: Supplementary_Measures, and Suppl. 5 for how to read it. Abbreviations:,,$+-=$ : number of significant increases, decreases, and non-significant changes, compared to wake control; bb: broadband; ab: all bands; $\boldsymbol{\delta}$ : delta-band; $\boldsymbol{\theta}$ : theta-band; $\boldsymbol{\alpha}$ : alpha-band; $\boldsymbol{\beta}$ : beta-band; $\boldsymbol{\gamma}$ : gamma-band; DOC: disorders of consciousness; $\boldsymbol{C I}$ : consistency index (overall consistency of effect directionality compared to wakefulness, see section 2.2 and Suppl. 3); MLAEPi\&AAI: middle latency auditory evoked potential index and a-line autoregressive index; BIS: bispectral index; SpEn: spectral entropy; LZc: Lempel Ziv complexity; SEF95: spectral edge frequency 95\%; ApEn: approximate entropy; AEP: auditory evoked potential; N100: the negative evoked component at 100ms; PeEn: permutation entropy; SaEn: sample entropy; MI: mutual information; P3a: the positive evoked component at $\sim 300 \mathrm{~ms}$; PCI: perturbational complexity index; ASSR: auditory steady state response; PLI: phase lag index; TEP: transcranial magnetic stimulation evoked potential; DFA: detrended fluctuation analysis; GFS: global field synchrony; ERP: event-related potential category; TF: time-frequency category; SD: signal diversity category; C: connectivity category; HV: measure affected by high variance in between group classification; EMG: measure affected by muscle tone and activity; IFT: 
measure unable to discriminate response from non-response during isolated forearm technique; ROC: measure unable to capture return of consciousness after loss of consciousness; PG: measure unreliable for certain demographic or patient groups; DR: measure unable to discriminate between report of dream content and no report following awakening during sleep; O: other comment. 